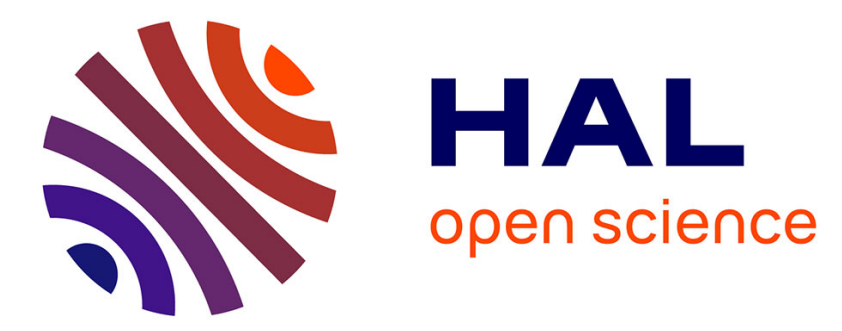

\title{
Agent-based system simulation of wireless battlefield networks
}

Younes Regragui, Najem Moussa

\section{To cite this version:}

Younes Regragui, Najem Moussa. Agent-based system simulation of wireless battlefield networks. Computers and Electrical Engineering, 2016, 56, pp.313-333. 10.1016/j.compeleceng.2016.07.007 . hal-01477111

\section{HAL Id: hal-01477111 \\ https://hal.science/hal-01477111}

Submitted on 27 Feb 2017

HAL is a multi-disciplinary open access archive for the deposit and dissemination of scientific research documents, whether they are published or not. The documents may come from teaching and research institutions in France or abroad, or from public or private research centers.
L'archive ouverte pluridisciplinaire $\mathbf{H A L}$, est destinée au dépôt et à la diffusion de documents scientifiques de niveau recherche, publiés ou non, émanant des établissements d'enseignement et de recherche français ou étrangers, des laboratoires publics ou privés. 


\title{
Agent-based system simulation of wireless battlefield networks
}

\author{
Younes Regragui ${ }^{\mathrm{a}}$, Najem Moussa ${ }^{\mathrm{a}, *}$ \\ ${ }^{a}$ LAROSERI, Department of Computer Science, Chouaib Doukkali University, El Jadida, Morocco.
}

\begin{abstract}
Mission-critical military operations with dismounted soldiers are frequently characterized by high battlefield dynamics. In such scenarios a mobility model can manage soldiers' movements dynamically especially under enemy attacks. This paper presents a Group Mobility Model simulating realistic soldier and leader battlefield behaviors. Our model analyzes communication between a group of dismounted soldiers deployed in a mobile ad hoc network (MANET) and their leader under several perturbation factors (e.g., noise and enemies attacks) which affect movements and topology connectivity. Results show that the dismounted soldiers' collective movement improves the capacity of communication channels, whereas noise uncertainty may dramatically destroy the network. Moreover, the enemy's presence, another disorder parameter, changes qualitatively and quantitatively the army's wireless communication topology. Enemy numbers decrease almost linearly the throughput at the sink node (commander). A discussion of results follows, using distributions of path lifetimes, path lengths, packet delivery and group sizes in the communication soldiers' network.
\end{abstract}

Keywords: Self-Organized Behavior, Mobility Models, Ad hoc Networks, Group mobility, Dismounted soldiers, Battlefield.

\section{Introduction}

Recently, the army has been interested in developing new skills and competencies such as making soldiers more connected [1] in the battlefield based on modern soldiers' electronic equipment and computer technologies by using mobile wireless ad hoc networks (MANETs) [2, 3]. The utilization of dismounted soldiers is one of the major strategies being adopted in the Army which makes tactical operations on the battlefield much easier to control. In a military environment, the dynamics of such missions may change rapidly, so the dismounted soldier would need to incorporate several new technologies to exchange information consisting of surveillance and tactical operations in order to prevent intrusion and detect enemies[2].

In the context of military operations, autonomous dismounted soldiers may interact simultaneously with the environment (battlefield) and with each other so as to complete an assigned mission such as a sweep operation of houses or buildings in a wide area, containing features such as mountains, forests, or rivers. The group of soldiers may be divided into a number of battalions with each one having its own mission, especially in some critical situation (e.g., searching and attacking the enemies during a sweep operation or escaping from an unexpected enemy attack). This stressful overcharge of interactions, in conjunction with the geographically variable nature of the battlefield area and the unpredictable behavior in terms of wireless network topology state, thus increasing the susceptibility of network topology to decomposition in multiple components.

The network connectivity is worsened due to topology changes driven by wireless links and unpredictable mobility of soldiers. The highly dynamic in nature of dismounted soldiers' mobility on the battlefield and node failures are considered among the main reasons for this challenging problem. Therefore, the evaluation of the network performance during the execution of a tactical military scenario for such situations in the

\footnotetext{
*Corresponding author: N. Moussa

Email addresses: regragui.y@ucd.ac.ma (Younes Regragui), moussa.n@ucd.ac.ma (Najem Moussa)
} 


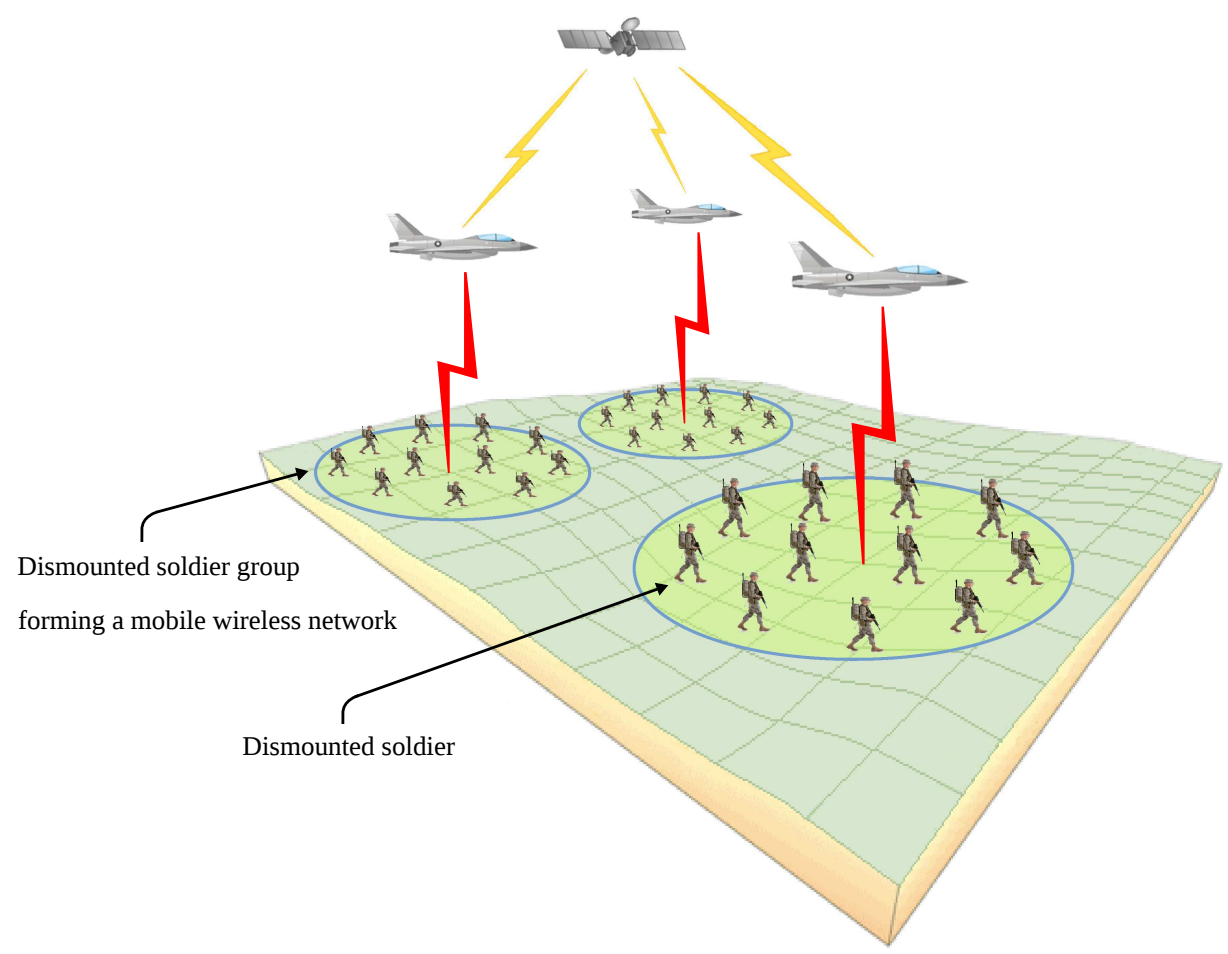

Fig. 1. Illustration of dismounted soldier group in military modern communications infrastructure.

real world setting is in many cases not feasible since the cost can be too high and it is impossible to test in real world deployment of soldiers in the battlefield with the presence of enemies. Therefore, a simulation environment is very attractive for evaluating and studying the impact of soldiers' behaviors on the performance of the network and topology condition during military operations. Typically, the utilization of a simulation environment is intended to reflect and assess real-world scenarios accurately such as modeling the mobility and wireless communication of soldiers on the battlefield as realistically as possible. After the deployment of soldiers on the battlefield, the destination direction of each soldier is done via a mobility model in the simulation, including the velocity of each one and their interaction with one other.

In the literature, various group mobility models have been proposed for studying and simulation of MANET in a real-world scenario. Among them the reference point group mobility (RPGM)[4] and Group force mobility model (GFMM), which are the most commonly used group mobility models to simulate the battlefield scenarios. In [5] the authors focus on group interaction and movement, but also provides the capability to incorporate obstacles into a simulation area and give the groups the ability to maneuver around them. However, in most of the existing group mobility models, the authors did not focus explicitly on more complex situations such as investigating the effect of enemies' attacks on the battlefield. They focused in the most cases on communication in battlefields with the presence of geometric obstacles in the form of buildings or inter-vehicular communication. To the best of our knowledge, this study is the first one that proposes a modeling approach for a military scenario based on the presence of enemies on the battlefield in mobile ad hoc networks (MANETs).

In summary, the aim of this paper is to introduce a group mobility model to simulate wireless communication on the battlefield of a dismounted soldier group with and without the presence of enemy attacks as realistically as possible. For our purposes, we were interested in studying our model as follows:

- Evaluation of network performance of dismounted soldiers with the presence of perturbations factors (noise) used as a flexible modeling of obstacles. 
- Network performance analysis in terms of tactical dynamics of dismounted soldiers on the battlefield in the presence of enemies. Moreover, we provide statistical evaluations based on the connectivity of soldier groups on the battlefield, especially when soldiers are trying to escape from enemy attacks in which unexpected network topology change may occur.

This paper introduces a group mobility model, in order to realize a performance study of a collective motion of dismounted soldiers in a MANET network with the commander (leader) defined as a sink node. This study is evaluated through various mobility scenarios and based on several metrics focusing on data throughput, path lifetime, packet loss, packets delivery ratio, the velocity of soldiers, etc. We perform NS-2 simulations to observe and analyze these network metrics. Here, the proposed model is implemented with tcl scripting language and works in Linux environment.

The rest of the paper is organized as follows. The related work is presented in Section 2. Section 3 describes the collective motion approach we have used in our group mobility model. Experimental results are presented in Section 4. Discussion and comparison of this work and other methods are carried out in Section 5. Section 6 concludes the paper and discusses the future research.

\section{Related work}

In this section, we will review Group mobility models which make efforts to simulate collective motion behaviors in real world situations. The second part of this section shows how nodes mobility can have the worst effect on the nature of the network topology and increasing data loss in the network; it also shows numerous solutions which have been proposed to this challenging issue.

\subsection{Group mobility models}

There have been many attempts to adopt group mobility models in cooperative tactical mobility and military ad hoc networks as the most suitable models to realistically model the mobility of nodes in MANETs. Group mobility models define how the mobile nodes distributed within a geographic area move as a group. Previously, several group mobility models based on a lead point have been proposed to simulate mobility in real world situations. The Reference Point Group Mobility Model (RPGM) [4] is the most widely known of the group mobility models. Each group has a logical "center" which defines the entire group's motion behavior, including location, speed, direction and acceleration. The trajectory of the logical center can be predefined or obtained based on a particular entity mobility model. In [6], the Reference Velocity Group Mobility Model (RVGM) extends the RPGM model by proposing two velocity vectors: group velocity of logical center or the lead point, and the local deviation velocity of each group member. In [5], the authors introduced the Group Force Mobility Model (GFMM) which is similar to RVGM in that the velocity of a group member follows the velocity of its lead point with a small random deviation. GFMM not only focuses on group interaction and movement but also provides the capability to incorporate obstacles into a simulation area and gives the groups the ability to maneuver around them. Authors in [7] proposed a Structured Group Mobility Model (SGMM), which parameterizes group structure and generates movement sequences for use in simulations. The SGMM uses reference point as in RPGM, which may be the geographical center of the group, the location of the leader, or the group's center of mass. In [8] the authors introduced a new group mobility model called the Diamond Group Mobility (DGM), where a group of soldiers is restricted in a diamond region with one dismounted soldier at the center considered as (commander). In Diamond Group Mobility, a dismounted soldier group has a logical center as in RPGM. The center's motion defines the entire group's motion behavior.

While these models assume all nodes move based on a lead point considered as a logical center that exists within each group, in other cases group mobility models are based on the location of the next region or area to which the mobile nodes group is moving. For example, in the Reference Region-Based Group Mobility (RRGM) [9], every group is associated with a reference region which is an area that nodes will move towards to and once they arrive, the nodes will move around within the region waiting for the arrival of other nodes. In [10], the authors proposed a virtual track based group mobility model (VT model) which closely approximates the mobility patterns in military MANET scenarios. It is reported that the proposed 
VT mobility model is suitable for both military and urban environment. In [11], the authors proposed a mobility model (Hierarchical Group Mobility model) designed for modeling a military operation in order to study the connectivity of a MANET established through wireless communication between the moving objects (ships, vehicles or foot soldiers, and aircraft). In a hierarchical mobility model, every moving object will be a child of another moving object, and the coordinate system of its movements will have the parent object's position as its origin.

In recent years, several group mobility models approaches have been proposed to improve the performance of collective movement in MANETs. In [12], an enhanced version of the Community Mobility Model was proposed which incorporates a feature that encourages group mobility. This model follows the preceding community-based approaches that map communities to a topological space.

In [13], in order to avoid intra-group and inter-group collision and also to avoid collision with environmental obstacles, the authors proposed a novel group mobility model for mobile ad hoc networks (MANETs), named Bird-Flocking Behavior Inspired Group Mobility Model (BFBIGM), which takes inspiration from the mobility of a flock of birds flying in a formation. In [14], the authors introduced STEPS, a generic and simple modeling framework for mobile opportunistic networks based on the principles of preferential attachment and location attractor. Furthermore, the STEPS Model is inspired by observable characteristics of the human mobility behavior, specifically the spatial-temporal correlation of human movements. In order to obtain meaningful performance results of real human movement simulation in MANETs, the authors in [15] proposed the N-body mobility model for wireless network research that is capable of synthesizing the group-forming tendency observed in real human movements. Combined with a clustered network generation algorithm, the N-body model does not require detailed knowledge of the target scenario, but rather synthesizes mobility traces for large populations based on metrics extracted from a small sample trace.

\subsection{Topology maintenance in (MANETs)}

Several methods have been proposed for link failure protection in ad hoc networks due to unpredictable nodes mobility in highly dynamic topology. Examples of these are, partition prediction and service replication on the server nodes [16] or data replication at multiple nodes and dynamically deploying these nodes to disconnected partitions of the network [17]. However, both data replication and topology information updates undoubtedly increase memory and communication bandwidth overhead. In [18], the authors proposed a distributed strategy based on the directional information, in which a node grows it transmission power until it finds a neighbor node in every direction. However, this strategy leads to a worse degradation of energy due to multiple attempts to change the transmission power level. In [19], a hybrid connectivity restoration framework (HERO) was presented by integrating the proposed connectivity restoration algorithm with a potential function based dynamic motion controller. HERO is able to restore the connectivity of mobile networks subjected to a single or multiple simultaneous agent failures.

Other works based on decentralized approaches are proposed to improve route recovery in MANET. The authors in [20] present a decentralized approach for maintaining the connectivity of a MANET using autonomous and intelligent agents. However, this approach requires an adaptive strategy to guide agent behaviors, for which the effectiveness depends highly on the density of the network. In [21], a particle swarm optimization (PSO)-based lifetime prediction algorithm for route recovery in MANET was proposed. This technique predicts the lifetime of link and node in the available bandwidth based on parameters such as the relative mobility of nodes and energy drain rate, etc. Each particle contains a local memory space to store the best position experienced by the particle until then. Using this information, the velocity of the particle can be estimated. Using predictions, the parameters are fuzzified and fuzzy rules have been formed to decide on the node status.

Based on the drawbacks of previous works, in [22], a backbone based multicast routing protocol was proposed to recover the link failures in MANETs. This protocol is a hybrid protocol which combines the features of both trees-based and mesh based routing techniques.

Another class of approaches is based on using backup paths to reduce node failure and link failure in MANETs. For example, in [23], to discover efficient stable communication channels with longer lifetimes and an increased number of packets delivered, a multi-path routing protocol was proposed to protect intermediate nodes of the path instead of just the links between two neighboring nodes. In [24], several recovery 
mechanisms were proposed to reduce the impact of failure. In this work, the author compute the backup route through a routing table to alleviate the impact of this on the network by finding an alternative path ready to use to pass the traffic when the primary one fails.

In the current research, we analyze the effect of the collective motion of soldiers on the performance of a military wireless network in a simulation approach. Then, we propose different performance metrics in order to give more attention to network topology change during the mobility of soldiers in the battlefield. In addition, we analyze the effect of collective motion on the performance of the network with the presence of enemies in the battlefield. As far as we know, the present paper is the first work dealing with simulation of the collective motion of a soldier group with the presence of enemies in the wireless communication model on the battlefield.

\section{Dismounted soldiers collective motion approach}

This section presents the basic concept of our proposed group mobility model, describing how the movement of dismounted soldiers and enemies is modeled on the battlefield based on a set of simple rules. It illustrates how the superposition of these simple rules is used to govern the dynamics of autonomous agents belonging to two distinct groups (soldiers and enemies). The most important consideration of the group mobility model in trying to simulate the self-organizing behaviors of dismounted soldiers and enemies is due to the fact that this model is the most appropriate one for modeling and simulation of mobility on the battlefield area $[4,10,11]$. In this paper, we assume that dismounted soldiers are equipped with wireless communication devices based on IEEE 802.11 and are able to communicate directly with the soldier leader or indirectly via multi-hop routing.

\subsection{The collective motion behavior}

Collective motion is a self-organized behavior of independent agents. Many categories of social cohesion and animals' aggregation behavior are found in nature (e.g. ant colonies, birds flock flying and fish swarms, soldiers in the battlefield) carry out their tasks collectively in order to contribute to a common goal. Even though individuals cooperate to accomplish a given global complex mission (e.g. foraging, migration, nest building, defense against enemies in the battlefield, etc.), an individual has only a local perception of the surrounding environment and displays specific behavioral tendencies which are governed by using a few simple rules. In addition, [25, 26] introduced three basic rules.

- cohesion: attempt to stay close to each other.

- separation: behavior that avoids collisions by causing a soldier to steer away from all of its neighbors.

- alignment: behavior that causes a particular soldier to line up with soldiers close by.

In $[25,26]$, to maintain a flock cohesion, the agent is able to make independent decisions and interacts with a fixed number of neighbors, rather than with all neighbors in the flock. In [26], the authors demonstrated that individuals in a bird flock can change their position relative to others based only on local information.

In this article, we proposed a group mobility model based on a collective motion approach for military wireless communications on the battlefield, where a group of dismounted soldiers moves in a limited battlefield area. A potential field algorithm is used to generate movement for each soldier. The perceptual field of each dismounted soldier is divided into the zone of repulsion ( $\mathrm{ZoR}$ ), the zone of orientation ( $\mathrm{ZoO})$ and the zone of attraction (ZoA), as shown in Fig. 2. Each dismounted soldier attempts to maintain a minimum distance from others within the ZoR. Within the ZoO, a dismounted soldier aligns itself with its neighbors and within the ZoA, a dismounted soldier moves towards the group so as not to be on the periphery or be left behind. Soldiers cannot see too far, thus, there is no interaction with others located outside the ZoA.

The proposed strategy of collective motion in this article is similar to the rule-based process in Couzin's model [26] and Reynolds's model [25]. However, in order to model the interaction of soldiers and enemies, we need to define some new behavioral rules assigned to soldiers when detecting enemy attacks and vice-versa (see subsection 3.1). 


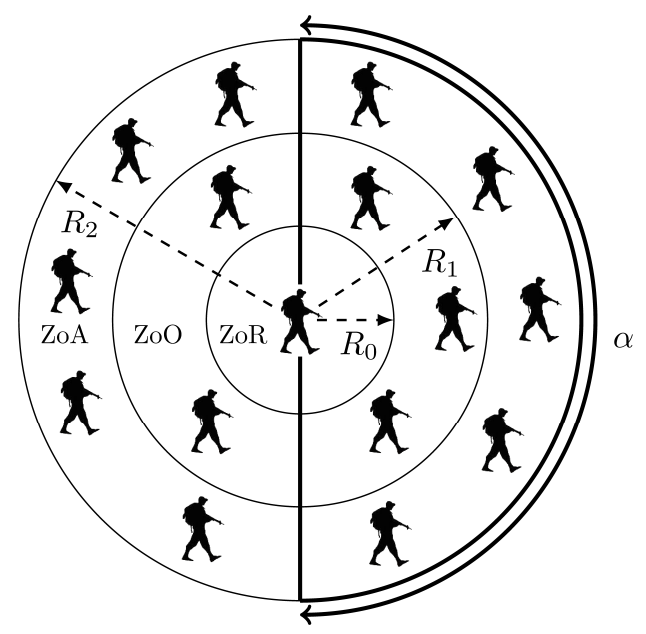

Fig. 2. Representation of a member in the model centered at the origin: $z o r=$ zone of repulsion, zoo $=$ zone of orientation, zoa=zone of attraction, zore=zone of repulsion from enemies, $\alpha=$ field of perception ahead of the member.

\subsection{Behavioral rules assigned to dismounted soldiers and enemies}

\subsubsection{Behavioral rules assigned to dismounted soldiers}

1) A Dismounted soldier attempts to maintain a maximum distance between himself and the enemies in his neighborhood, at all times regardless of his location zone. This rule has the highest priority.

2) The leader dismounted soldier attempts to move in any direction if there aren't enemies in his neighborhood.

3) If the dismounted soldier is not performing any previous rules, he tends to maintain a minimum distance between himself and the others soldiers within the zone of repulsion. This rule has also the highest priority (less than rule 1 or rule 2 ).

4) If the dismounted soldier is not performing rule 3 he tends to align himself with the leader dismounted soldier within the zone of orientation $(\mathrm{ZoO})$, and towards the position of the leader dismounted soldier within the zone of attraction (ZoA).

5) If the leader dismounted soldier is neither in $\mathrm{ZoO}$ nor in $\mathrm{ZoA}$, the dismounted soldier tends to align himself with his neighbors within the zone of orientation $(\mathrm{ZoO})$, and towards the group within the zone of attraction $(\mathrm{ZoA})$.

\subsubsection{Behavioral rules assigned to enemies}

1) The enemy leader attempts to move towards leader soldier, whenever he is within his neighborhood, in order to attack the soldiers group.

2) The enemy attempts to attack soldiers if there are one or more soldiers in his neighborhood, regardless of his location zone. For that, the enemy moves towards the group of soldiers within his neighborhood. This rule has the highest priority for enemies.

3) If an enemy is not performing any previous rules, he tends to maintain a minimum distance between himself and the others enemies within the zone of repulsion. This rule has also the highest priority (less than rule 1 or rule 2 ).

4) If the enemy is not performing rule 3 , he tends to align himself with the leader enemy within the zone of orientation( $\mathrm{ZoO})$, and towards the position of the leader enemy within the zone of attraction (ZoA).

5) If the leader enemy is neither in $\mathrm{ZoO}$ nor in $\mathrm{ZoA}$, the enemy tends to align himself with his neighbors within the zone of orientation( $\mathrm{ZoO})$, and towards the group within the zone of attraction (ZoA). 


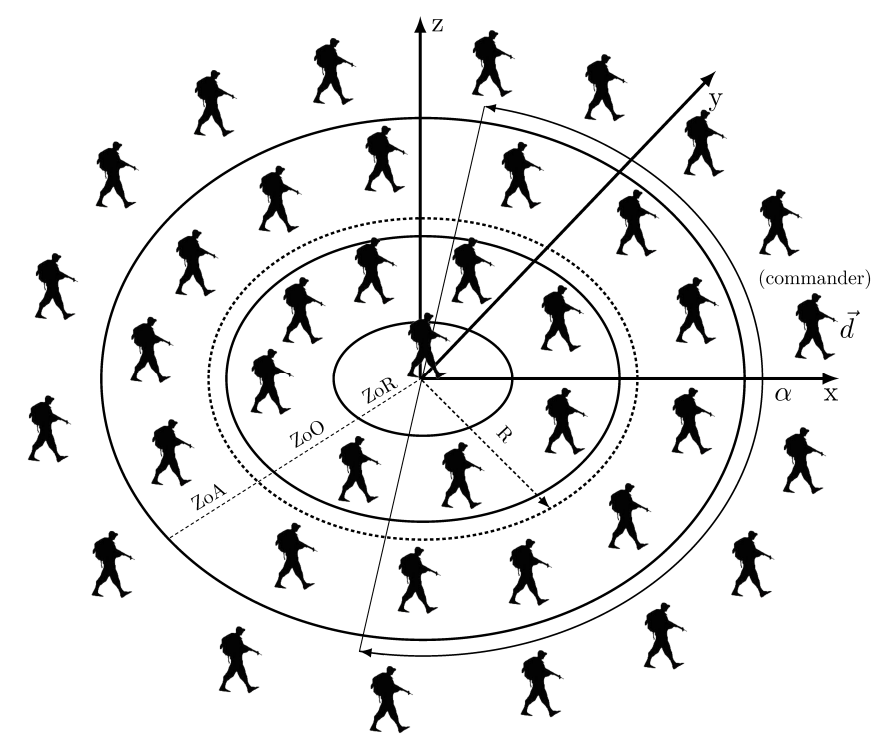

Fig. 3. Representation of the area around a dismounted soldier placed in the center: ZoR is the zone of repulsion, $\mathrm{ZoO}$ is the zone of orientation and $\mathrm{ZoA}$ is the zone of attraction. $\alpha$ degrees is the field of view, $\mathrm{R}$ is the communication range, $\vec{d}$ is the direction of movement for commander member (leader soldier).

\subsection{Behavioral rules description}

A simulation model of $N$ soldiers and $M$ enemies was created for which we assume that individuals move at a constant speed of $v_{0}$ units per second. Each soldier is characterized by his location $\mathbf{p}_{i}(t)$ and velocity $\mathbf{v}_{i}(t)=v_{0} \times \mathbf{d}_{i}(t)$ of direction $\mathbf{d}_{i}(t)$ at time $\mathrm{t}$. In each time step $t$, a member $i$ assesses the position and/or orientation of neighbors in its local neighborhood within three non-overlapping behavioral zones (Fig. 2) to determine its desired direction of motion $\mathbf{d}_{i}(t+d t)$ at time $t+d t$. After that, the member $i$ turns towards the direction vector $\mathbf{d}_{i}(t+d t)$ by the turning angle $\alpha_{i}$, where

$$
\alpha_{i}=\varphi+\xi_{i}
$$

where $\varphi=\sigma \times d t$ is the turning angle and $\sigma$ is the turning rate. $\xi_{i}=\theta \times d t \times \operatorname{rand}(0,1)$, is a random uncertainty variable and $\theta$ is a noise parameter. The location of the member $i$ at time $t+d t$ is given by:

$$
\mathbf{p}_{i}(t+d t)=\mathbf{p}_{i}(t)+\mathbf{v}_{i}(t+d t) d t
$$

In addition, introducing the uncertainty in the movement of the military group may be useful as a means of analysis of the effects of various factors on the battlefield such as crossing in difficult terrains (mountains, forests, and rivers), surveying a military region or buildings from enemies.

\subsection{Behavioral rules description of a dismounted soldier}

\subsubsection{Repulsion behavior from enemies}

Each dismounted soldier $i$ (this may be the leader soldier) attempts to maintain maximum distance from $n_{e n}$ enemies in its neighborhood regardless of their location zone. In most cases, enemies are detected once they are within the zone of attraction $(\mathrm{ZoA})$ of the dismounted soldier $i$. The direction of repulsion from enemies is given as follows:

$$
\mathbf{d}_{r e}(t+d t)=-\sum_{j=1}^{n_{e n}} \frac{\mathbf{r}_{i j}}{\left|\mathbf{r}_{i j}\right|}
$$


where $\mathbf{r}_{i j}$ is the unit vector from the location point of $i$ in the direction of the enemy $j$. This behavioral rule has the highest priority in the model, so that if $n_{e n}>0$, the desired direction $\mathbf{d}_{i}(t+d t)=\mathbf{d}_{r e}(t+d t)$. This repulsion behavior can be interpreted as soldiers avoiding danger space, or attacks from enemies. If no enemies are within any zone, the dismounted soldier responds to other behavioral rules.

\subsubsection{Repulsion behavior from neighbors}

A dismounted soldier $i$ attempts to maintain a minimum distance from the others soldiers within a zone of repulsion (ZoR), modeled as a circle, centered on the dismounted soldier $i$, with radius $R_{0}$. If $n_{r}$ neighbors are present in this zone at time $t$, the direction of repulsion from neighbors is given as follows:

$$
\mathbf{d}_{r}(t+d t)=-\sum_{j \neq i}^{n_{r}} \frac{\mathbf{r}_{i j}}{\left|\mathbf{r}_{i j}\right|}
$$

This behavioral rule has a priority compared to other behaviors, but, less than repulsion from enemies behavior priority. This zone can be interpreted as soldiers maintaining personal space, or avoiding collisions. Moreover, this repulsion behavior corresponds to a frequently observed behavior of animals in nature (Krause and Ruxton, 2002)[26]. If no neighbors are within the zone of repulsion $\left(n_{r}=0\right)$, the dismounted soldier responds to others rules within the zone of orientation $(\mathrm{ZoO})$ and the zone of attraction $(\mathrm{ZoA})$.

\subsubsection{Cohesion behavior}

Cohesion behavior is the opposite of repulsion behavior. This behavior encourages a dismounted soldier to move closer to other neighbors. The cohesion direction of a dismounted soldier $i$ is given as follows:

$$
\mathbf{d}_{i}(t+d t)= \begin{cases}\frac{1}{2}\left[\mathbf{d}_{o}(t+d t)+\mathbf{d}_{a}(t+d t)\right] & n_{o}>=1, n_{a}>=1 \\ \mathbf{d}_{o}(t+d t) & n_{o}>=1, n_{a}=0 \\ \mathbf{d}_{a}(t+d t) & n_{a}>=1, n_{o}=0\end{cases}
$$

where $n_{o}$ and $n_{a}$ are the number of neighbors in the zone of orientation (ZoO) and attraction (ZoA) respectively, $\mathbf{d}_{o}(t+d t)$ is the direction of alignment with neighbors within the zone of orientation $(\mathrm{ZoO})$, and $\mathbf{d}_{a}(t+d t)$ is the direction of attraction towards the positions of soldiers within the zone of attraction $(Z o A)$. The widths of zones (ZoO) and (ZoA) are defined as $\Delta r_{o}=r_{o}-r_{r}$ and $\Delta r_{a}=r_{a}-r_{o}$. Both $r_{o}$ and $r_{a}$ are used to determine the zones boundaries respectively. The cohesion direction is given as follows:

$$
\begin{aligned}
& \mathbf{d}_{o}(t+d t)=\sum_{j=1}^{n_{o}} \frac{\mathbf{v}_{j}}{\left|\mathbf{v}_{j}\right|} \\
& \mathbf{d}_{a}(t+d t)=\sum_{j \neq i}^{n_{a}} \frac{\mathbf{r}_{i j}}{\left|\mathbf{r}_{i j}\right|}
\end{aligned}
$$

\subsection{Behavioral rules description of an enemy}

\subsubsection{Enemy attacks}

In the strategy attack of the enemies, that is considered in this paper, the leader enemy attempts to move towards the leader soldier, whenever he is within his neighborhood, in order to attack the soldiers group. Moreover, an enemy $i$ attempts to attack soldiers if there are one or more soldiers in his neighborhood, regardless of his location zone. For that, the enemy moves towards the group of soldiers within his neighborhood. In the simulations, we suppose that this neighborhood is limited by the zone of attraction (ZoA). Hence, if $n_{\text {sol }}$ soldiers are within ZoA (centered at the position of the enemy $i$ ), the direction of attack is given as follows:

$$
\mathbf{d}_{a t}(t+d t)=\sum_{j=1}^{n_{s o l}} \frac{\mathbf{r}_{i j}}{\left|\mathbf{r}_{i j}\right|}
$$


Table 1. Simulation configuration.

\begin{tabular}{lllll}
\hline Parameter & Symbol & Value & Parameter & Value \\
\hline Number of soldiers & $\mathrm{N}$ & 50 & Simulation area & $5000 \times 5000 \mathrm{~m}^{2}$ \\
Enemy numbers & $\eta$ & $4-52$ & Transmission range & $80 \mathrm{~m}$ \\
Zone of repulsion & $r_{r}$ & $10 \mathrm{~m}$ & Propagation model & TwoRayGround \\
Zone of orientation & $\Delta r_{o}\left(r_{o}-r_{r}\right)$ & $40 \mathrm{~m}$ & Interface queue model & PriQueue \\
Zone of attraction & $\Delta r_{a}\left(r_{a}-r_{o}\right)$ & $150 \mathrm{~m}$ & Queue size & 64 \\
Turning rate & $\sigma$ & 0.1 & Routing protocol & AODV \\
Noise & $\theta$ & {$[0,1]$} & Transport protocol & UDP \\
Data generation rate & $\lambda$ & {$[0,1]$} & Packet generator & CBR \\
Initial velocity of nodes & $v_{0}$ & $1(\mathrm{~m} / \mathrm{s})$ & Packet size & 1000 bytes \\
\hline
\end{tabular}

where $\mathbf{r}_{i j}$ is the unit vector from the location point of $i$ in the direction of the dismounted soldier $j$. This behavioral rule has the highest priority in the model, so that if $n_{\text {sol }}>0$, then the desired direction $\mathbf{d}_{i}(t+d t)=\mathbf{d}_{a t}(t+d t)$. If no soldiers are within ZoA, the enemy responds to other behavioral rules.

\subsubsection{Repulsion and Cohesion behaviors for the enemies}

The repulsion behavior from neighbors (enemies) and the cohesion behavior are identical with those of the soldiers due to fact that these behavioral rules govern individual-level interactions within their group.

After the above process has been performed for every member (soldier and enemy), all members move towards the desired direction $d_{i}(t+d t)$ at time $(t+d t)$ by a vector velocity $\mathbf{v}_{i}(t+d t)$. We apply this process at each time step $d t$, where each member is able to independently perform a specific rule according to the significant interaction with other members in its neighborhood.

\section{Simulation analysis}

\subsection{Performance Metrics}

Here we define several metrics related to the performance of the network measurement. We monitor the change in topology status caused by the motion of soldiers in the battlefield with and without the enemy' presence. Our main concern is the dynamic nature of the network topology and its effect on the network performance. The measured performance metrics are described in details as follows.

- Metric 1: The throughput, defined as the average rate of successful packets delivery over a communication channel to sink node.

- Metric 2: The forwarded throughput, defined as the average rate of successfully forwarded packets through intermediate nodes over a communication channel.

- Metric 3: The packet loss, defined as the total dropped packets by source or intermediate nodes during transmission.

- Metric 4: The system speed, $V_{a}$, defined as the average velocity of soldiers during the simulation, can be calculated as:

$$
V_{a}=\frac{1}{N v_{0}}\left|\sum_{i=1}^{N} \mathbf{v}_{i}(t)\right|
$$

where $\mathrm{N}$ is the number of soldiers in the battlefield, and $v_{0}$ is the initial velocity assigned to soldiers at the beginning of the simulation execution.

- Metric 5: The path lifetime indicates how long the path is still valid before receiving a path update or path error message. 
- Metric 6: The path length, defined as the total number of hops traveled by the packet to reach the destination.

- Metric 7: The packet delivery ratio, defined as the number of successfully received packets at the destination to the total number of packets which are expected to be received at the destination.

- Metric 8: The group size: defined as the number of soldiers who are geographically isolated from others by a distance $d_{i g}{ }^{1}$ and share a membership in the group.

\subsection{Analysis of the model without presence of enemies}

Our network contained $\mathrm{N}$ mobile nodes/soldiers deployed close to each other in a simulation area of $5000 \times 5000 \mathrm{~m}^{2}$. The movement pattern is based on a collective motion approach. At each time step $d t^{2}$, the soldiers/nodes are able to send data packets towards the sink node/commander via AODV routing protocol with a data generation rate $(\lambda)$.

\subsection{Effects of data generation rate $\lambda$}

Fig. 4a-b show the effect of data generation rate on both the average throughput and the average packet loss with varying values of noise $\theta$. The figures show that the throughput gradually increases as $\lambda$ increases. It is quite reasonable according to the network bandwidth availability.
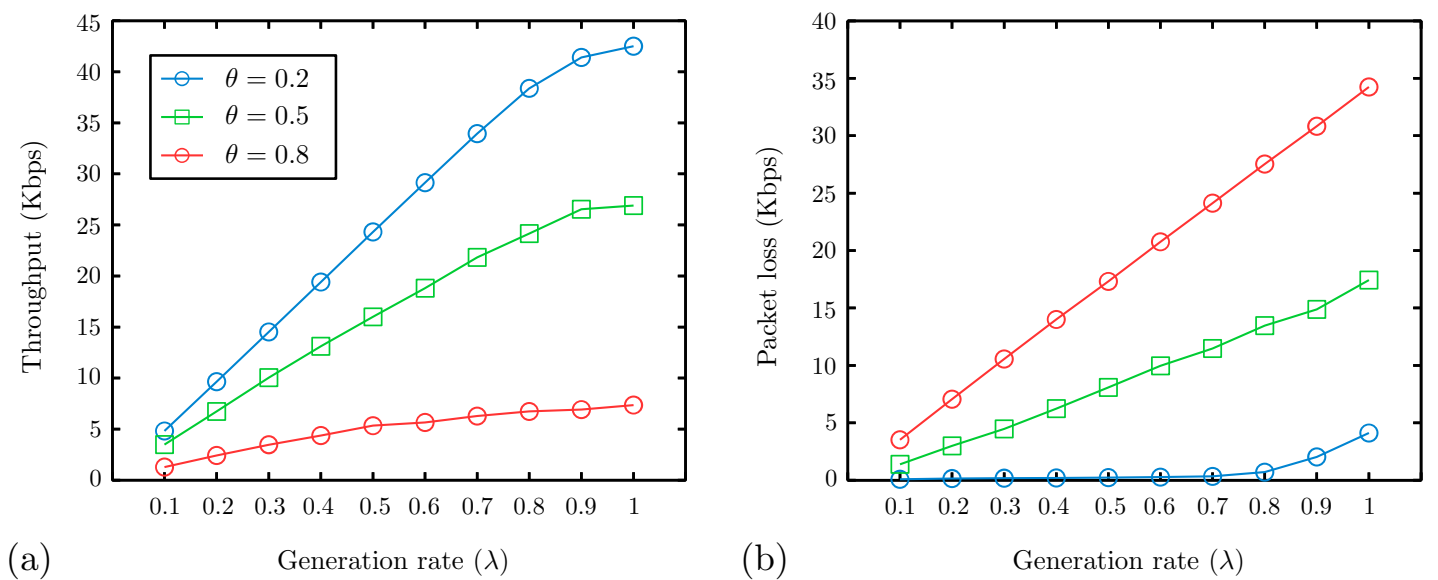

Fig. 4. Experiment 1: Effect of increasing the generation rate $(\lambda)$ with parameters $v_{0}=1(\mathrm{~m} / \mathrm{s}):(\theta)$ the noise probability parameter.

On the contrary, the noise has an obvious impact on the throughput, i.e., it decreases drastically in proportion to the noise. The reason for this is that noise exerts a perturbation behavior on the mobility of soldiers. Therefore, topology change has a great impact on the routing protocol performance. As opposed to the throughput, packet loss is taken into account. Fig. $4 \mathrm{~b}$ shows that the average packet loss increases significantly with $\lambda$, because of buffer-overflow and collisions due to network congestion. Furthermore, noise strongly affects the packet loss rate. The reason for this is that noise exerts an influence on the stability of links, by causing an unpredictable mobility behavior, which has the direct effect of increasing the packet loss, due to link failures and indirectly on the increasing buffer overflow because of unreachable nodes. In the next subsection, we will extend the analysis to noise effect on different network performance metrics to investigate how noise $(\theta)$ can affect on collective motion of soldiers and network topology, and then its impact on inter-node communication within the network resultant topology.

\footnotetext{
${ }^{1}$ The distance between two isolated groups $d_{i g}=80 \mathrm{~m}$.

${ }^{2}$ The time step $d t$ equals 1 second.
} 


\subsection{Effects of noise $\theta$}

It has been demonstrated that collective motion models [27] exhibit a phase transition which occurs when the noise is increased. Indeed, for small values of noise, the average system speed is approximatively equal to one (see Fig. 5c). This phase which is called "finite net transport phase" corresponds to a coherently moving phase where almost all nodes move with the same direction. However, for high values of noise, this mean velocity is approximately zero; reflecting the random aspect of the directions of the moving nodes. This phase is called, "no transport phase". Hence, a phase transition from "finite net transport phase" to "no transport phase" occurs at some critical value $\theta_{c} \approx 0.6$ (see Fig. $5 \mathrm{c}$ ).
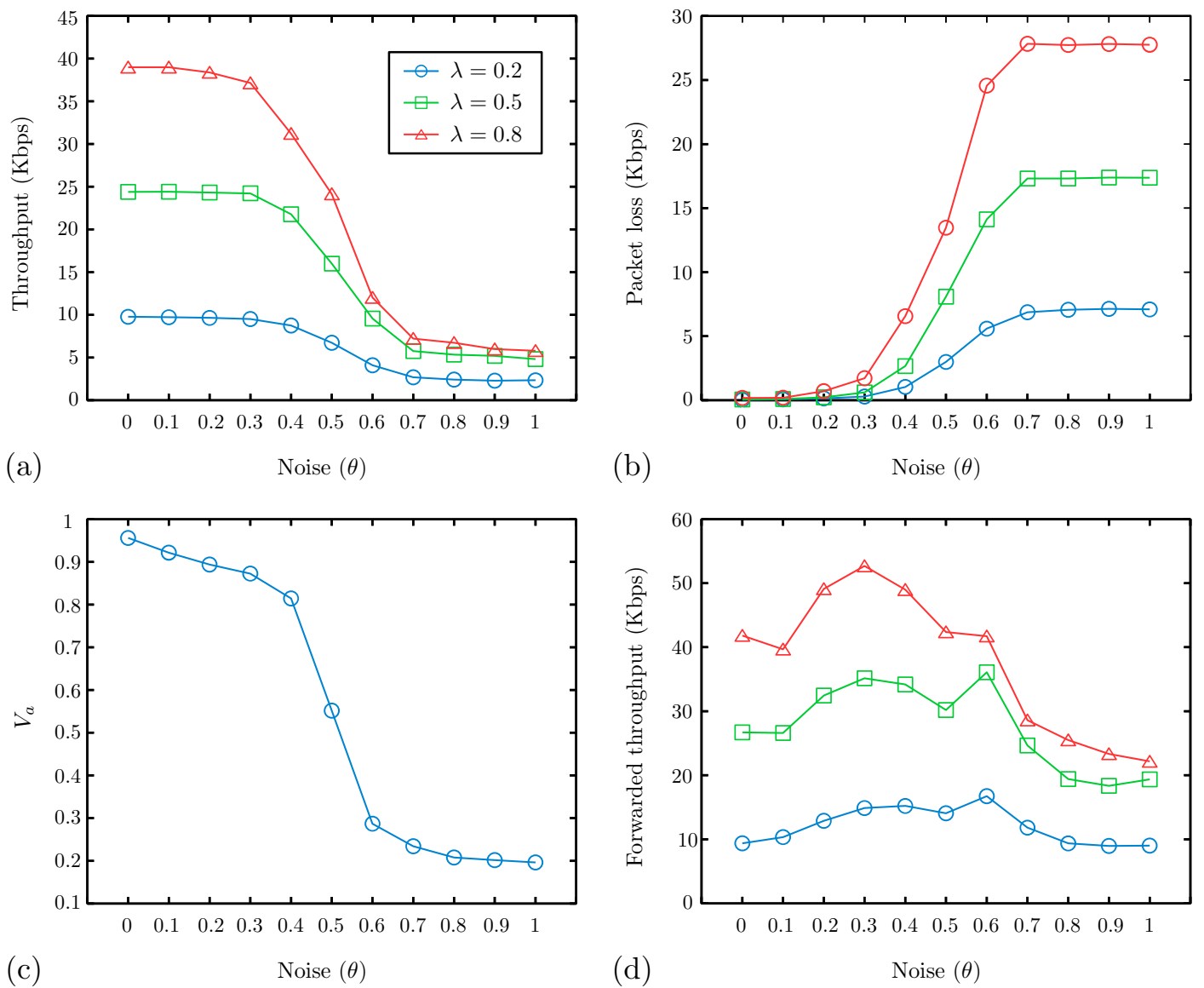

Fig. 5. Effect of increasing noise $\theta$ with parameters $v_{0}=1(\mathrm{~m} / \mathrm{s})$ : (a) Average throughput, (b) Average packet loss, (c) Average velocity, and (d) Average forwarded throughput.

From Fig. 5a, we see that the average throughput decreases significantly versus noise. Hence, the throughput remains almost constant in the finite net transport phase; while it decreases rapidly towards small values in a no transport phase. The main effect on decreasing throughput results especially from increasing noise, which hardly causes topology change due mainly to perturbation of the collective motion model. Thus, an arbitrary partition of the network occurs when noise increases.

Fig. 5b illustrates the effect of noise on average packet loss. It is found that the packets will begin to be removed from the network when the noise exceeds a certain level. Beyond this value, the network enters into the congested phase. The results show also that under highest noise, packet loss is high, due mainly to bad condition of network topology where the link failures rate is very important.

In wireless mobile networks, nodes move frequently and to cover the disconnected segments of the net- 
work, nodes may act as a router to forward packets to other nodes. In order to study the influence of noise on the appearance of relay nodes in the network, we show in Fig. 5d the average forwarded throughput versus noise under different generation rates values $\lambda$. The average forwarded throughput significantly increases with increasing noise until reaching a maximized value and then decreases at higher values of noise. If the noise value is very small, then all the nodes move closer to each other and there is no need for relay nodes to transfer the data to the sink node. Moreover, increasing noise will provoke a considerable dispersion of nodes in the network area and then lead to the appearance of long paths and link failures, whereas direct transmission may be insufficient to reach the destination. Hence, this will increase the appearance of rely nodes in the system. However, if the noise value is very high, the nodes become almost disconnected and then no communications could be achieved via relay nodes. This explains the decreasing of the forward throughput (see Fig. 5d).

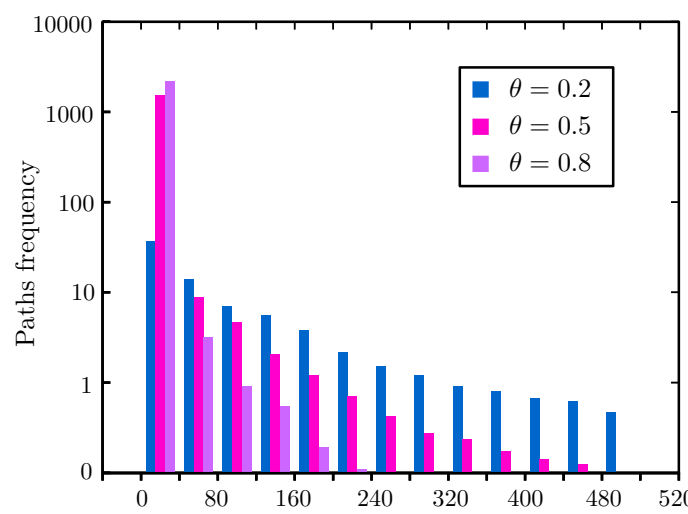

(a)

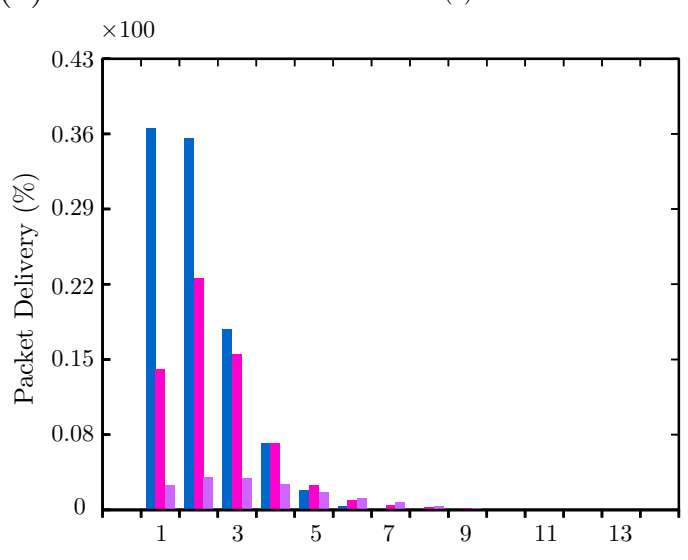

(c)
Hop Length

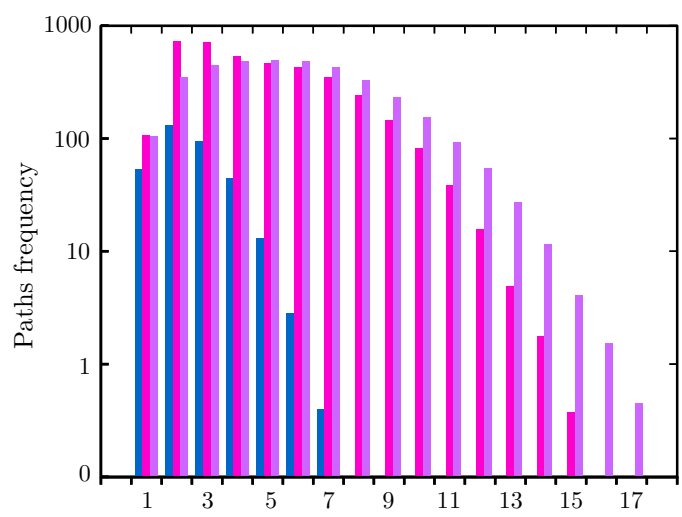

(b)

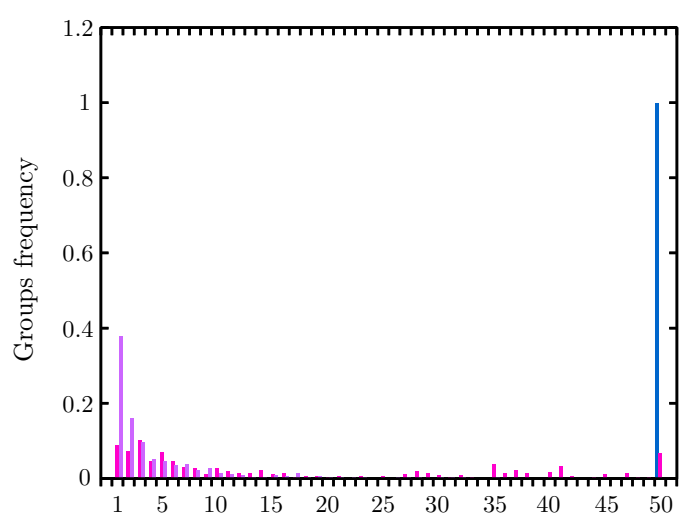

(d)

Group size

Fig. 6. Effect of increasing noise $\theta$ with parameters $v_{0}=1(\mathrm{~m} / \mathrm{s})$, and $\lambda=0.8$ : (a) Average path lifetime, (b) Average path length, (c) packet delivery ratio, and (d) Group frequency

Fig. 6a-b illustrate the distributions of the path lifetime and the path length in the communication soldiers network. The results show that for higher values of noise, paths with the shortest lifetimes are the most prevalent in the network (see Fig. 6a). The reason for this is that both the commander and the soldiers move separately in a disordered fashion. Thus, smaller path lifetimes are due principally to the incapability of nodes to keep connectivity for a long time because of high dispersion of nodes. Furthermore, we see clearly from Fig. 6b that high values of noise lead to the establishment of long paths in order to reach the destination. However, these paths are frequently perturbed by unexpected movements of soldiers 
in the battlefield. As a consequence, this situation will lead to a significant reduction of the capacity of communication between the soldiers and leader soldier in the battlefield (see Fig. 5a).

Fig. $6 \mathrm{c}$ reports the performance of the network obtained in terms of packet delivery ratio under different values of $\theta$. Fig. $6 \mathrm{c}$ shows that the network achieves an overall higher rate of received packets under lower values of noise. The reason for this is that under the low value of noise, soldiers move coherently in a collective motion structure (finite net transport phase) and then the communication paths are more stable and reliable. Therefore, most existing communication paths are those emanating from soldiers that are a single hop from a commander. Furthermore, under a low noise values, all nodes are located close to each other. So, obviously, there is no need for establishing longer paths. This optimizes the capacity of the overall communication network. But, under a higher values of noise, the network is segmented into several groups sizes which are changing frequently over time (see Fig. 6d).
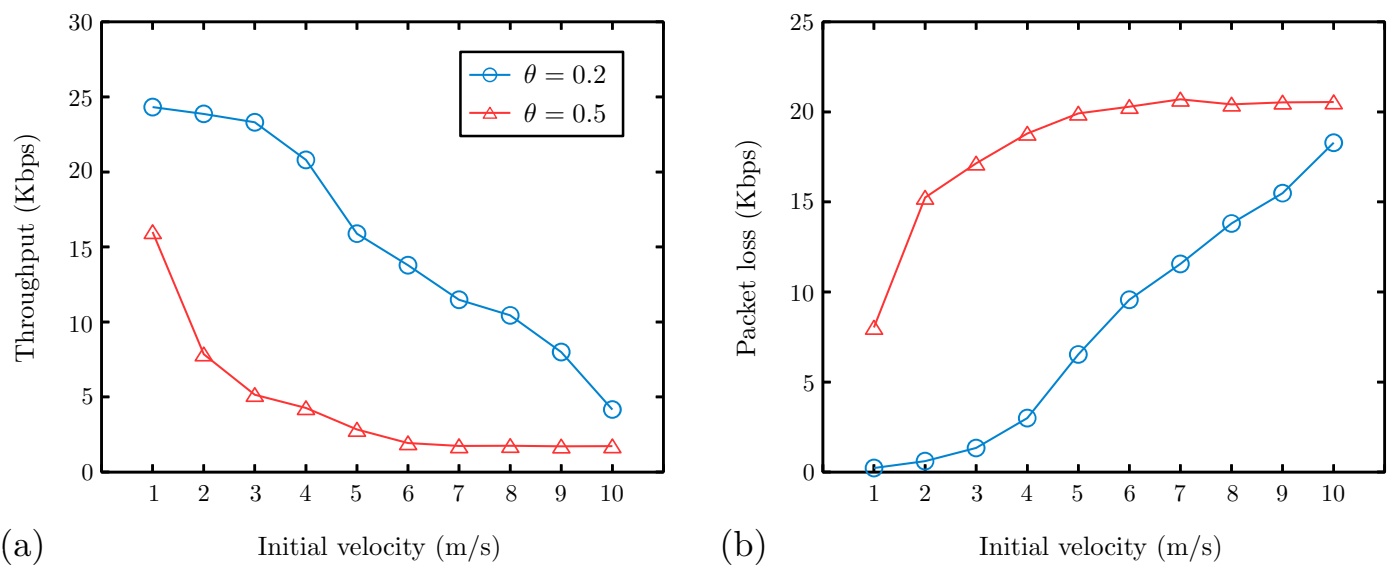

(a)

Initial velocity $(\mathrm{m} / \mathrm{s})$

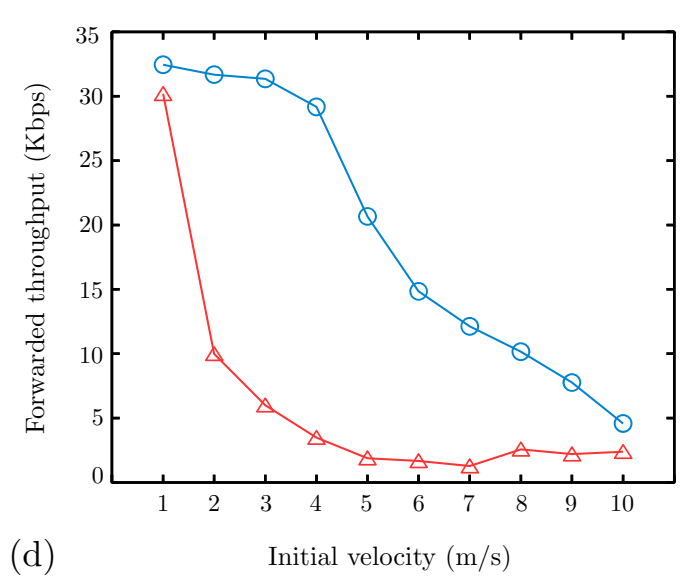

(c)

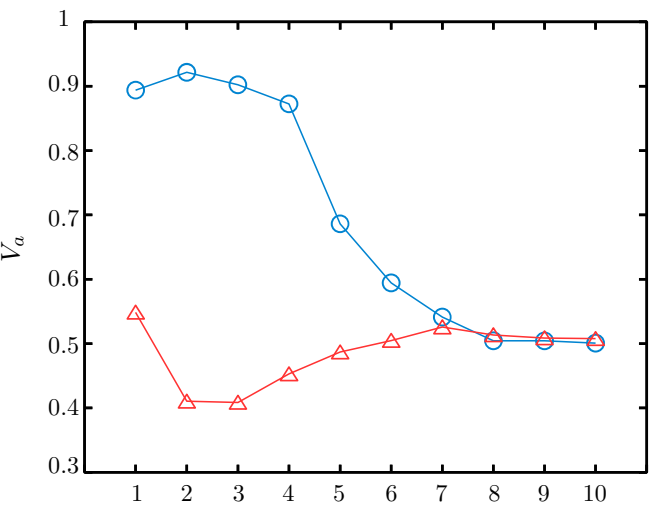

Initial velocity $(\mathrm{m} / \mathrm{s})$

(d)

Fig. 7. Effect of increasing initial velocity $v_{0}$ with parameters $\lambda=0.5$ : (a) Average throughput, (b) Average packet loss, (c) Average velocity of soldiers, and (d) Group frequency.

Hence, we see that at low values of noise, only one large group exists in the network. However, increasing noise may create the dispersion of nodes and then several groups of different sizes may appear in the system. In particular, high frequency of disconnected nodes is present in the network. Thus, this segmentation into different groups has a direct effect on the degradation of network performance as seen from (Fig. 5a and Fig. 6c). 


\subsection{Effects of initial velocity $v_{0}$}

We assume that our network will be strongly affected by the initial velocity of agents, whereby the communication efficiency of agents may be reduced; and especially when associated with the increase of noise, in which case agents may unexpectedly lose connections with their neighborhood due to the relatively quick change in the direction of the velocity vector of the agents. It has been shown that if $v_{0}$ goes to infinity, the agents become completely mixed between two states, similar to the mean-field behavior of a ferromagnetic [27]. The first state corresponds to perfect alignment of the group while the second corresponds to a pure random state. Therefore, we expect that the average velocity of the group will be exactly equal to 0.5 . While extreme of $v_{0}$ equaling zero, the agents are stationary where the agents do not move.

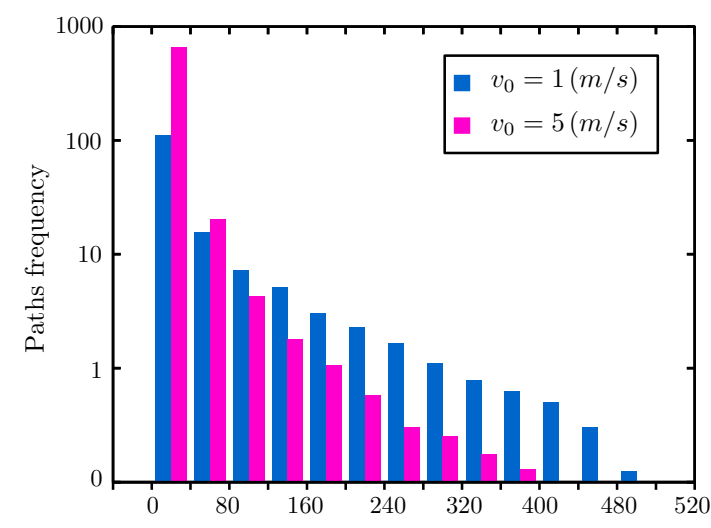

(a)

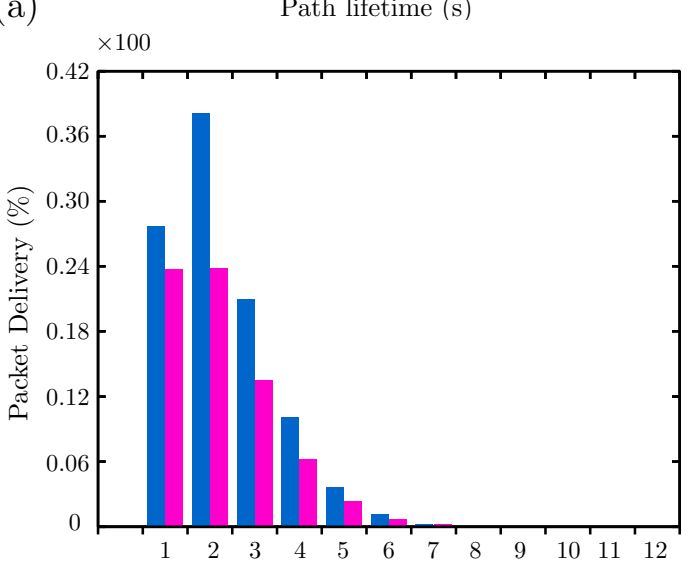

(c)

Hop Length

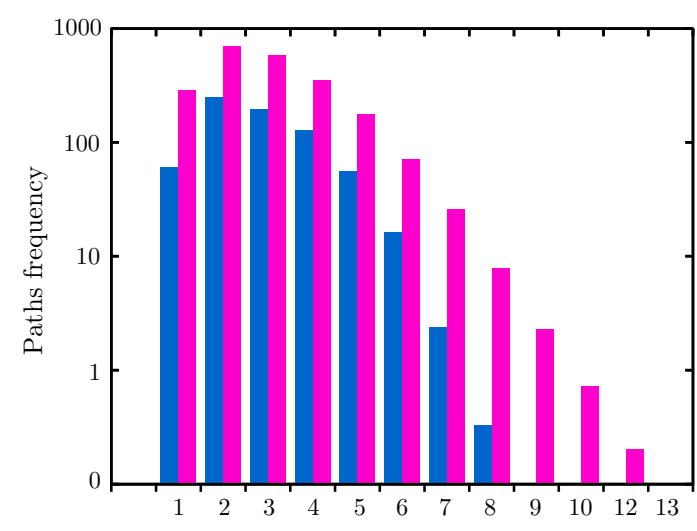

(b)

path length

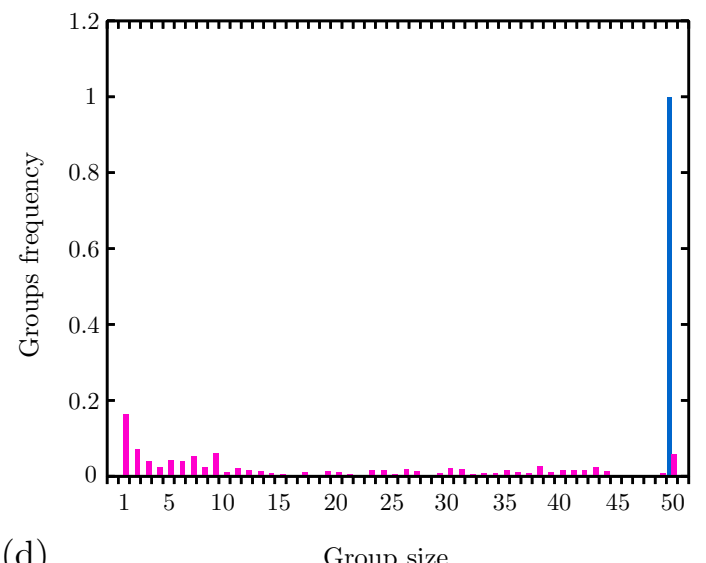

(d)

Group size

Fig. 8. Effect of increasing initial velocity $v_{0}$ with parameters $\theta=0.2$, and $\lambda=0.5$ : (a) Average path lifetime, (b) Average path length, (c) packet delivery ratio, and (d) Group frequency.

To evaluate the effects of the mobility on the wireless network of soldiers, we analyzed, with simulations, the effect of initial velocity $v_{0}$ on different performances metrics with varying values of $\theta=\{0.2,0.5\}$. The results of our simulation show that for $\theta=0.2$, increasing the initial velocity $v_{0}$ will affect slightly the mean velocity until it reaches $v_{0} \approx 4$ (see Fig. $7 \mathrm{c}$ ). But beyond this value, it decreases strongly until reaches the value 0.5 for $v_{0} \geq 8$. However, for $\theta=0.5$, we found that the mean velocity decreases until it reaches a minimum and then increases until reaches the value 0.5. Moreover, just as it was predicted, we find that for higher values of $v_{0}$, the mean velocity remains constant at the value 0.5 regardless of noise value.

Fig. 7a-b show that for lowest initial velocities, the network performs better, in terms of average through- 
put and packet loss, because low velocities provide favorable conditions that are sufficient for establishing very stable paths and also avoid network partition. On the contrary, it produces worse results under highest initial velocities because high velocities cause sudden and severe disruptions to ongoing network routing; resulting in a lower throughput and high packet loss.

Fig. 7d shows that, for low values of noise, the forwarded throughput is decreased gradually when increasing initial velocity, while, in the case of high noise, it decreases exponentially. The impact of increasing both the initial velocity of soldiers and the noise provokes a drastic dispersion of the network topology, leading to a segmentation of the network topology into several small groups sizes or even into isolated nodes.

Fig. 8 plots the performance of the network, in the case of low noise value, in terms of the distributions of paths lifetimes, path length, hop length and group size. Fig. 8a-b show how the established paths can be affected by different values of $v_{0}$ (i.e., the initial velocity). We can see that when $v_{0}$ is low, communication channels of long lifetimes and short path lengths persist in the network. In addition, the appearance of communication channels of long lifetimes is principally linked to collective motion of nodes in the network.

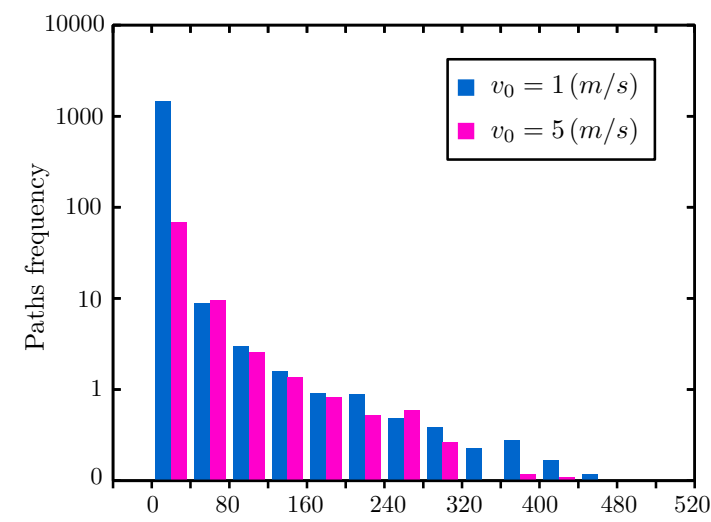

(a)

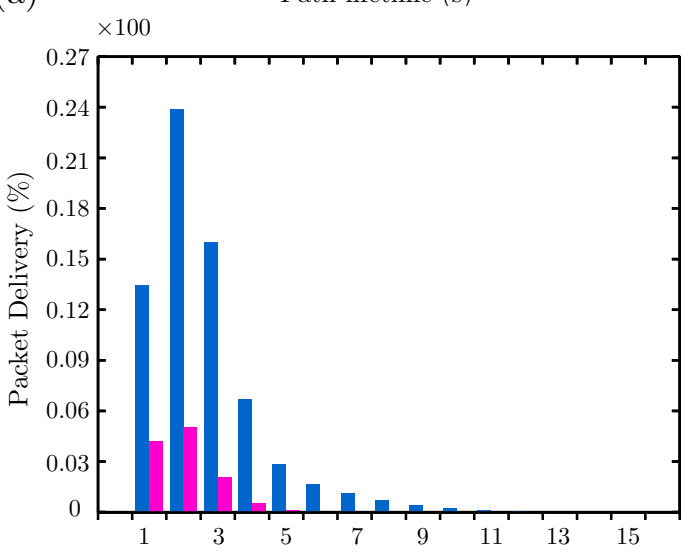

(c)
Hop Length

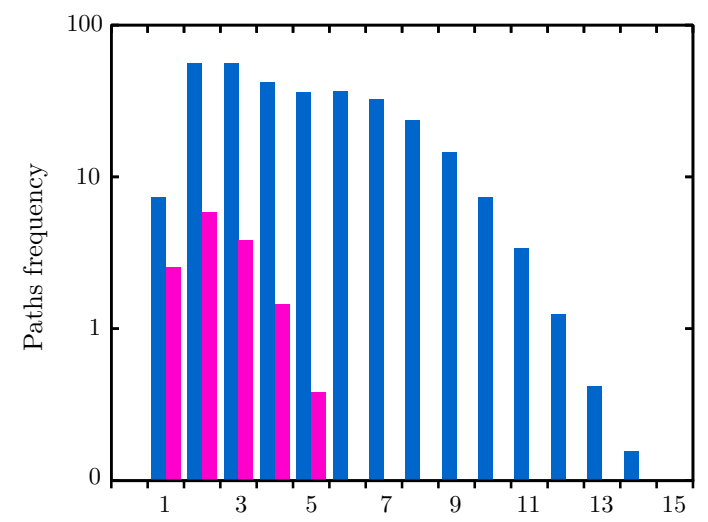

(b)

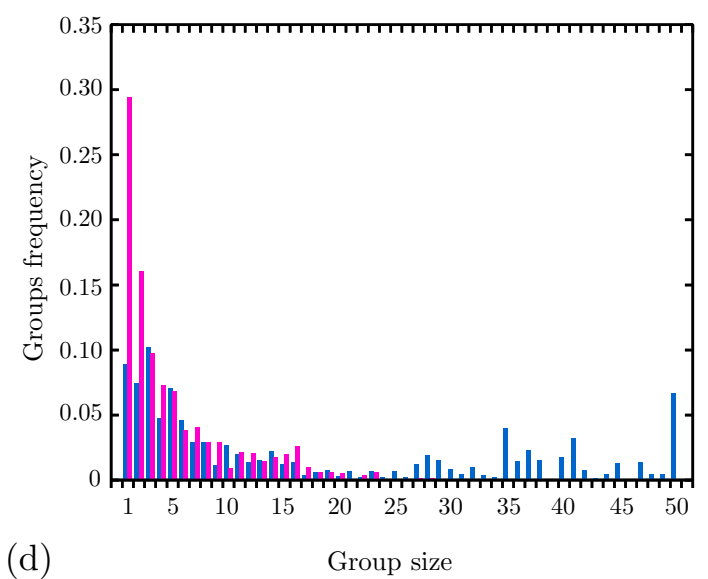

Fig. 9. Effect of increasing initial velocity $v_{0}$ with parameters $\theta=0.5$, and $\lambda=0.5$ : (a) Average path lifetime, (b) Average path lifetime, (c) Average connection lifetime, and (d) Groups frequency.

This can be seen clearly from Fig. 8d where only one large group exists in the system. However, increasing the mobility causes the appearance of a high number of established paths and most of them have short lifetimes. Thus, nodes aren't able to keep connectivity for a long time. Indeed, higher mobility will disperse the nodes into small groups, and data can be transferred to the sink only through communication channels 
having long paths. This confirms well the instability of the network due to a frequent topology change, where a lot of paths should be created in order to conduct packets towards the commander. Hence, much overheads is required to send traffic from the soldiers to the commander; affecting badly the network efficiency. To achieve better network efficiency, the soldiers must move slower in order to maintain the stability of their network. Indeed, we can see from Fig. $8 \mathrm{c}$ that when the initial velocity is low, packet delivery rate is improved and a higher percentage of packets are received by the commander through short paths.
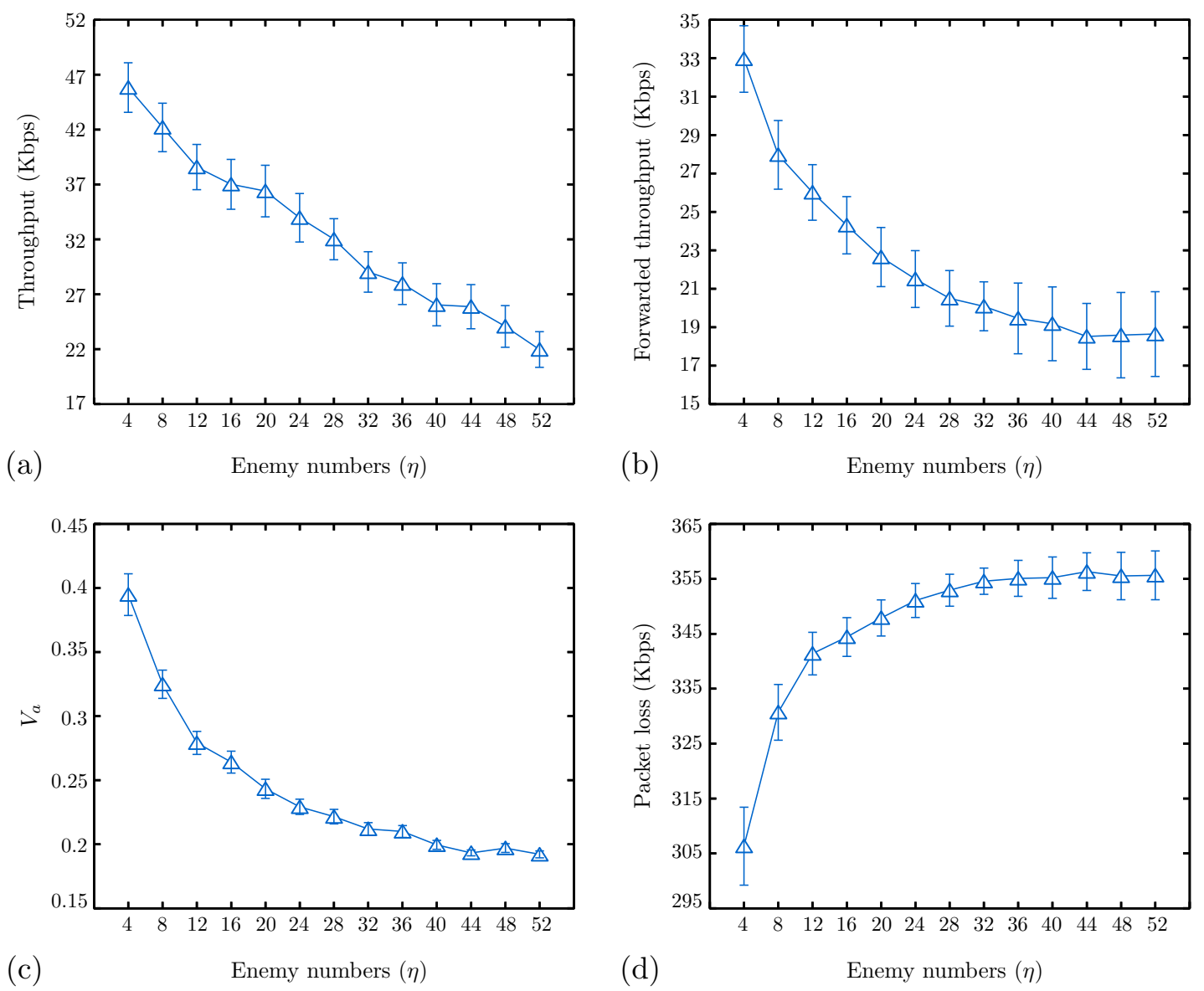

Fig. 10. Effect of increasing the enemy numbers $\eta$ in the battlefield with parameters $\theta=0, \lambda=0.5$, and $v_{0}=1(\mathrm{~m} / \mathrm{s})$ : (a) Average throughput, (b) Average forwarded throughput, (c) Average velocity, and (d) Average packet packet loss.

Moreover, to investigate the effect of initial velocity associated with a high level of noise, we repeated the same network scenario and calculations with different initial velocities and for $\theta=0.5$. This decision may highlight some features concerning the relationship between initial velocity and noise. We see from Fig. 9 that, for $v_{0}=1$ and $\theta=0.5$, the distribution of path lifetimes consistently shows a greater number of shortest lifetime paths compared with results in the case of $v_{0}=1$ and $\theta=0.2$ (see Fig. 9a). This is obvious since, as we have depicted before, noise invokes randomness and perturbations to the structure of the communication network. More interestingly, we show that when both the initial velocity and noise are very high, the nodes are rarely able to establish paths to the destination, due mainly to the high uncertainty of nodes' mobility within the network area (see Fig. 9b). In addition, we can expect that the association of higher levels of initial velocity and noise expands greatly the problem of the network partition and then causes severe topology destruction (see Fig. 9d). This can be seen clearly from experiment results shown in 
Fig. 9c, where the network efficiency is almost absent.

\subsection{Analysis of the model with the presence of enemies}

In this part, we use the same previous network configuration and the same group of soldiers, but, we add a varied enemy numbers in the battlefield near the group of soldiers. We assume that the enemy's leadership is able to move towards the soldier's leadership based on Military-intelligence, whereas the soldier's leadership is able to move randomly in the battlefield area. Furthermore, soldiers and enemies must necessarily follow the collective motion pattern separately. But, the enemies can attack soldiers in the case when an enemy remarks one or more soldier's existence in their field of view. Accordingly, enemies can't establish communication with soldiers or forward data flow to soldiers.

We analyzed the effect of increasing the enemy numbers $(\eta)$ in the battlefield on different system metrics. In Fig. 12, we show snapshots of the model where we see clearly that increasing the enemy numbers disperse the collective motion of the soldiers.
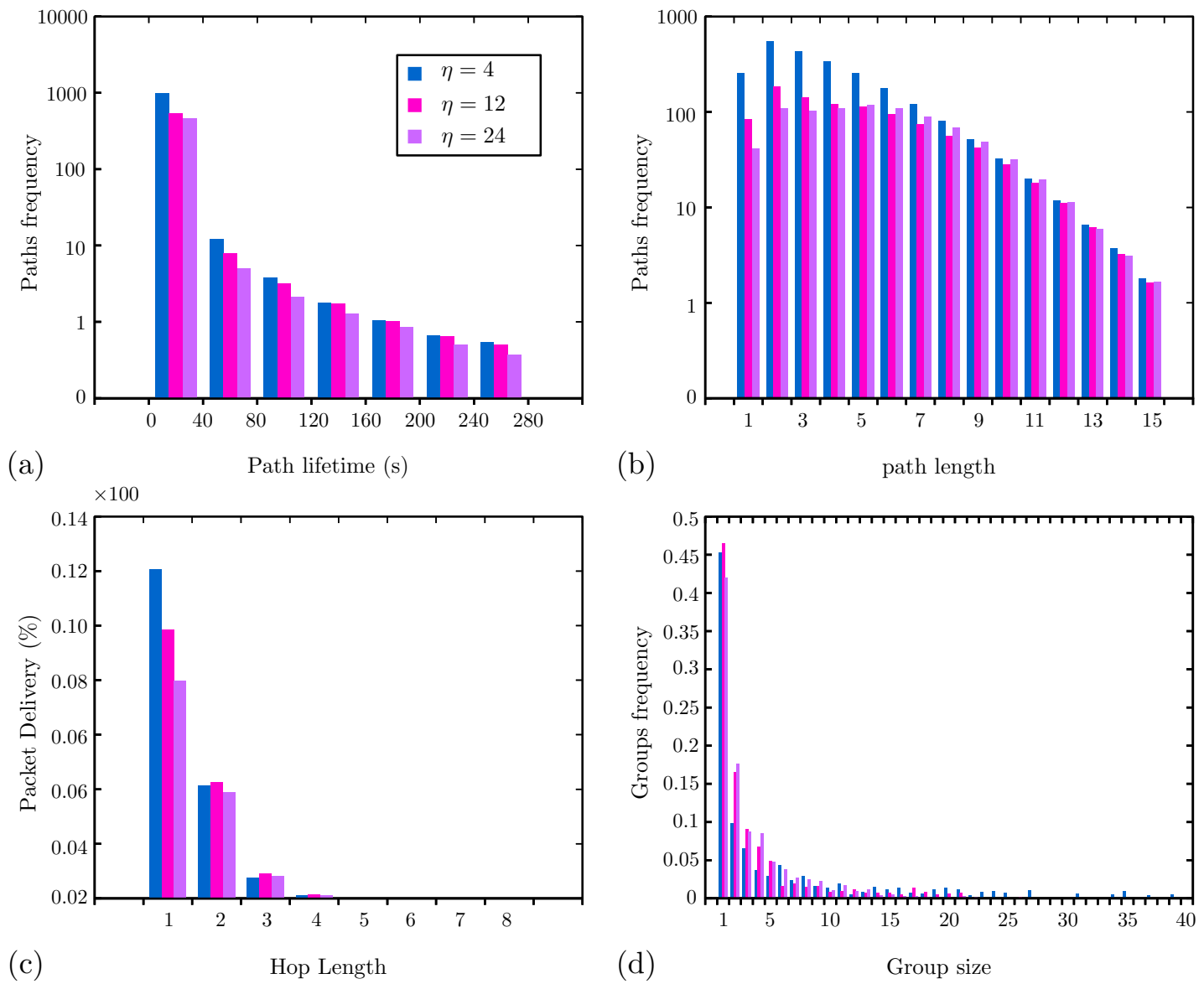

Fig. 11. Effect of increasing the enemy numbers $(\eta)$ in the battlefield with parameters $\theta=0, \lambda=0.5$, and $v_{0}=1(\mathrm{~m} / \mathrm{s})$ : (a) Average path lifetime, (b) Average path length, (c) Packet delivery count, and (d) Average groups size.

Fig. 10 shows that with increasing $\eta$, the average throughput at the sink node decreases almost linearly. It shows also that the average velocity exponentially decreased when increasing $\eta$ (Fig. 10a and Fig. 10c). Obviously, the presence of enemies in the battlefield provokes the dispersion of the group of soldiers in order 
to escape the enemy's attacks. This can be seen clearly from the distribution of the group size in the system Fig. 11d, where maximized percentage of isolated nodes are present.

In addition, we see that both the decreasing of the forwarded throughput and the increasing of the packet loss, are done in accordance with the average velocity variation (see Fig. 10b-d). Indeed, with increasing $\eta$, we observe an exponential decrease of the average velocity while, on the other hand, an exponential increases and an exponential decreases are found, for the forwarded throughput and the packet loss respectively. Moreover, even when the average velocity is stabilized for $\eta>=44$ they are also stabilized at the same value of $\eta$. Finally, we believe that the presence of enemies not only disperses the group of soldiers, but also destroys the communication network of the soldiers, so that the quantity of data flow transmitted to the commander decreases while increasing the enemy numbers.

Fig. 11 depicts the results of the distributions of path lifetimes, path lengths, hop lengths and group sizes under a different enemy numbers. Fig. 11a-b clearly show the appearance of a high number of long paths with short lifetimes in the wireless communication network of the soldiers. Thus, the presence of enemies in the battlefield causes a segmentation and disruption of the network topology and therefore causes a degradation of the network performance (see Fig. 11c). The enemy attacks are somewhat similar to the effect of noise parameter. However, the main difference lies in the fact that the enemy attacks are more realistic than the noise effect. While increasing the noise level decreases exponentially the throughput at the sink node, the increase of enemy numbers decreases proportionally the throughput. This implies that although the network may suffer from higher link failures and topology changes due to fact that soldiers are escaping from enemy. As a consequence, only the short paths may be established between the soldiers. Finally, the results show that the performance of the network was affected negatively by the presence of enemies in the battlefield. From these results, a high rate of dropped packets and low rate of packets delivery have been observed.

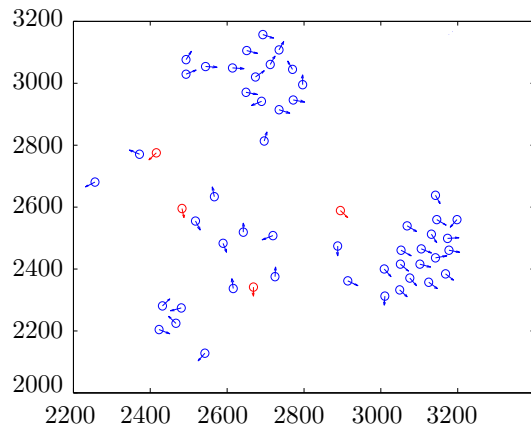

(a)

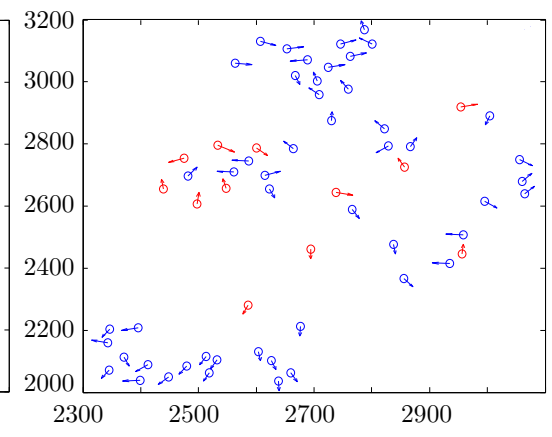

(b)

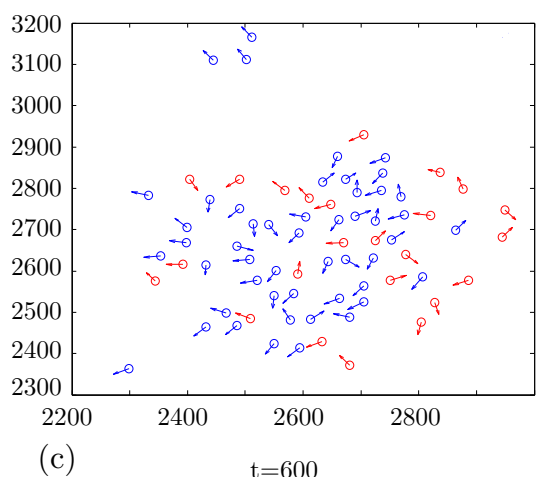

(c)

Fig. 12. Simulation with different enemy numbers $\eta$ in the battlefield with parameters $\theta=0, \lambda=0.8$, and $v_{0}=1(\mathrm{~m} / \mathrm{s}):$ (a) $\eta=4$, (b) $\eta=12$, and (c) $\eta=24$.

Indeed, in the presence of enemies, the highly dynamic and the sparse distribution of soldiers in the battlefield, lead to a decrease in path lifetimes and an increase in path lengths, which sharply degrades the network performance. Thus, there is a need to develop new infrastructures based on mobile ad hoc network capable of routing the urgent data of soldiers in the battlefield in a short time.

\section{Comparison with other existing mobility models}

Our study used several monitoring metrics to reveal the performance of our group mobility model in a complex environment (the battlefield). This model is designed to simulate the movement of dismounted soldiers with a leader on the battlefield. To quantify the reliability aspects and features of our model, a comparison with other mobility models is expected. Since, the simulation of enemy attacks is not treated in most of the existing group mobility models including recent works; only a comparison without the presence of enemies can be performed in this section. 
The unexpected mobility of soldiers in the battlefield leads to performance degradation of path length and path lifetime because speeding up or slowing down leads to changes in network topology. Comparing our model and other models in terms of path length and path lifetime is expected to give extra merit to their strengths and other features.

A performance comparison of our proposed Group Mobility Model along with some existing mobility models is provided for the perturbation factors of network topology similar to the noise effect defined in our model. Indeed, our mobility model was compared with RPGM[4], Nomadic[28], GFMM[5], STEMP ${ }^{3}[14]$ and $\mathrm{SLAW}^{4}[29]$. For this comparison, we have implemented the above mobility models with a scenario that consider a special node as the sink node (Commander) whereas the other members are considered as senders (Soldiers). In order to provide the randomness and perturbations to the structure of the network topology of existing mobility models as provided in our model via the noise parameter $(\theta)$, we identified through simulation the most appropriate parameters which could provoke a perturbation of nodes mobility for each mobility model as follows:

- Reference Point Group Mobility Model (RPGM): In each group, every member moves to a randomly chosen location within a circular neighborhood of radius $\mathrm{R}$ around its reference point location. The movement around the reference point is based on the Random Waypoint Model. The analysis and the simulation experiments demonstrated that the greater the radius the more the fluctuation and the uncertainty in the mobility is important.

- Nomadic Community Mobility Model: This is similar to RPGM where every member moves also to a randomly chosen location within a circular neighborhood of radius $\mathrm{R}$ around its reference point location. Nomadic is considered as a special case of the RPGM model.

- Group Force Mobility Model (GFMM): This supports the same logic design of the noise parameter which is defined as a speed deviation ratio. This parameter is used to control the deviation range of speeds of a node from the Group speed under GFMM.

- Spatio-Temporal Parametric Stepping (STEPS): A simple parametric mobility model which is inspired by observable characteristics of the human mobility behavior, specifically the spatio-temporal correlation of human movements. It supports an attractor power parameter named $(\alpha)$. We are interested in the variation of $\alpha$ from low to high value, where under the highest value of $\alpha$, the nodes have a higher probability to stay close to each other and so the preferential zone plays the attraction role instead of a repulsion one.

- Self-similar Least Action Walk (SLAW): This expresses the human walking patterns based on synthetic mobility traces. Under SLAW, people would always randomly choose places to visit in a random order. These places are defined as a set of waypoints and the choices of the next places or destinations to visit are completely random. Simulation experiments have demonstrated that the increase in number of waypoints $(\beta)$ is a determinant factor of perturbation degree of network topology.

Fig. 13 shows the average throughput as well as the packet delivery ratio in terms of different perturbation factors of network topology. As can be observed from Fig. 13, our model, RPGM and Nomadic exhibit the highest throughput as well as having the highest packet delivery $(\approx 100 \%)$ under low value of noise and radius $\mathrm{R}$, whereas the other mobility models exhibit the worst performance results.

By looking at Fig. 14a and Fig. 15a, the benefit of long path lifetimes and the cohesion between soldiers could be seen clearly for our model, RPGM and Nomadic. Furthermore, the existence of long path lifetimes, in these three mobility models, is an indication that there is an important mobility coherence between soldiers. This may leads to a significant increase in the number of relaying packets and therefore to optimizing the use of network resources.

\footnotetext{
${ }^{3}$ At the beginning of each simulation, the set of nodes is deployed within an area of $500 \times 500 \mathrm{~m}^{2}$. As the value of $\alpha$ decreases, the nodes will be distributed over the whole area.

${ }^{4}$ SLAW was evaluated over a coverage area of $1000 \times 1000 \mathrm{~m}^{2}$.
} 

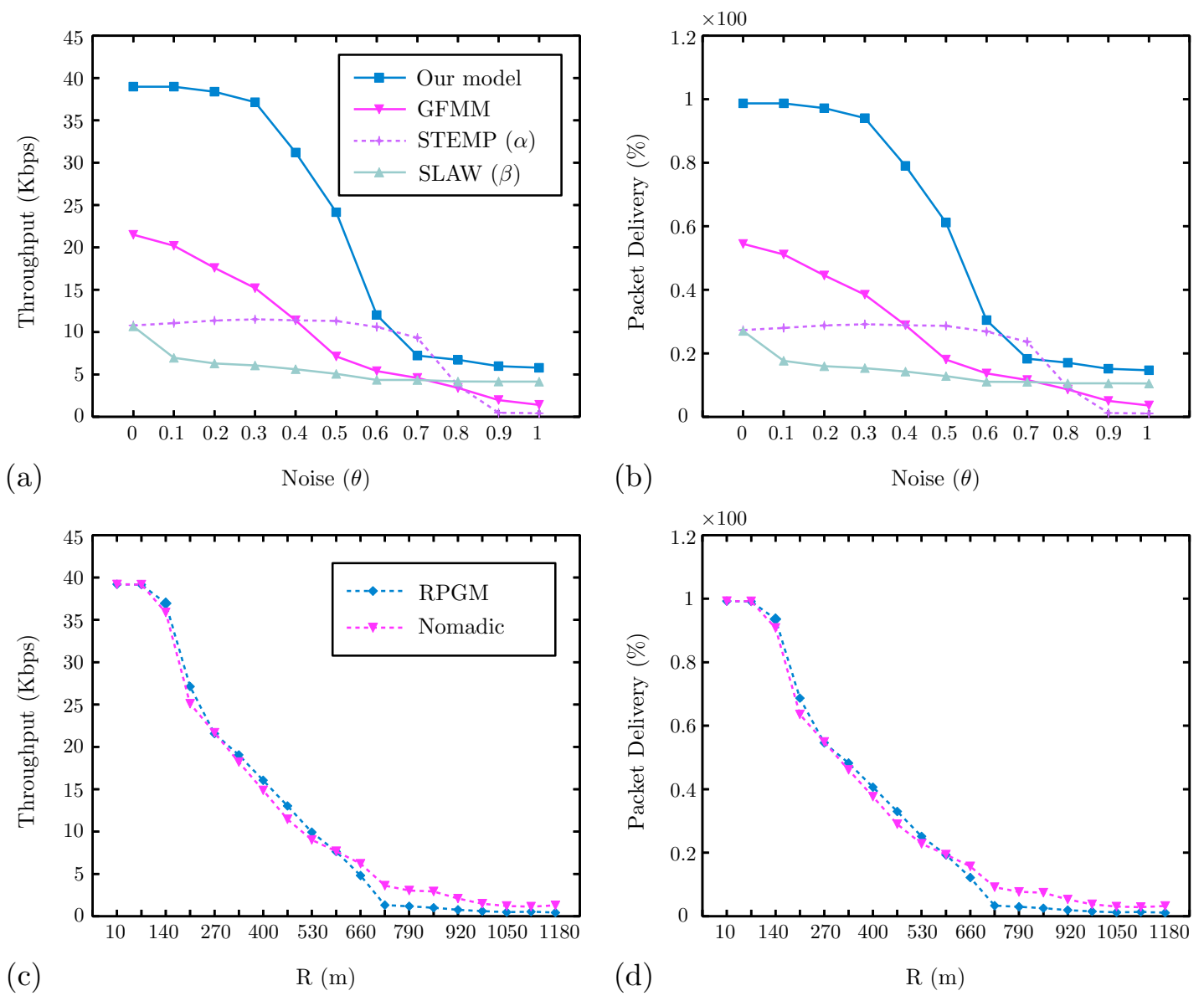

Fig. 13. Comparative evaluation between different mobility models in terms of the network performance, with parameters $v_{0}=1(\mathrm{~m} / \mathrm{s}), \lambda=0.8, \alpha=10 \times(1-\theta)$ and $\beta=(100 \times \theta)+10$.

Fig. 15a also shows that GFMM exhibits similar results in terms of group size compared with the three previous models. However in terms of network performance, it exhibits a medium level of throughput as well as the packet delivery ratio $(\approx 50 \%)$. This can be explained because nodes under GFMM are able to overlap or collide with each others; and then can generate collisions because of reception of high number of packets within a limited coverage area.

As the level of perturbation factors increases, the network performance exhibits a degradation for all models except for the STEMP model, where it shows a very slight increase in the network performance when $\alpha>=5$. However, when $\alpha$ is below the value of 5, indicating that the network in STEMP model becomes scattered, the nodes move in a highly unpredictable manner causing a segmentation of the network.

Observing the results presented in Fig. 13, Fig. 14 and Fig. 15, it is seen that the SLAW model depicts the worst network performance as $\beta$ increases. This can be explained because the mobility of nodes under the SLAW model is based on a purely random strategy where each node randomly tries to visit a selected waypoint from the total number of waypoints $(\beta)$. In this situation, the topology is quite unstable, and consequently the communication links between nodes will be unstable or may even become disconnected. On other hand, our model still exhibits the best performance compared to the other mobility models in terms of throughput, packet delivery ratio and path lifetime under medium value of noise. Fig. 15b shows clearly that the highly stable communications in our model are achieved by wide group distributions of nodes within the network area. 

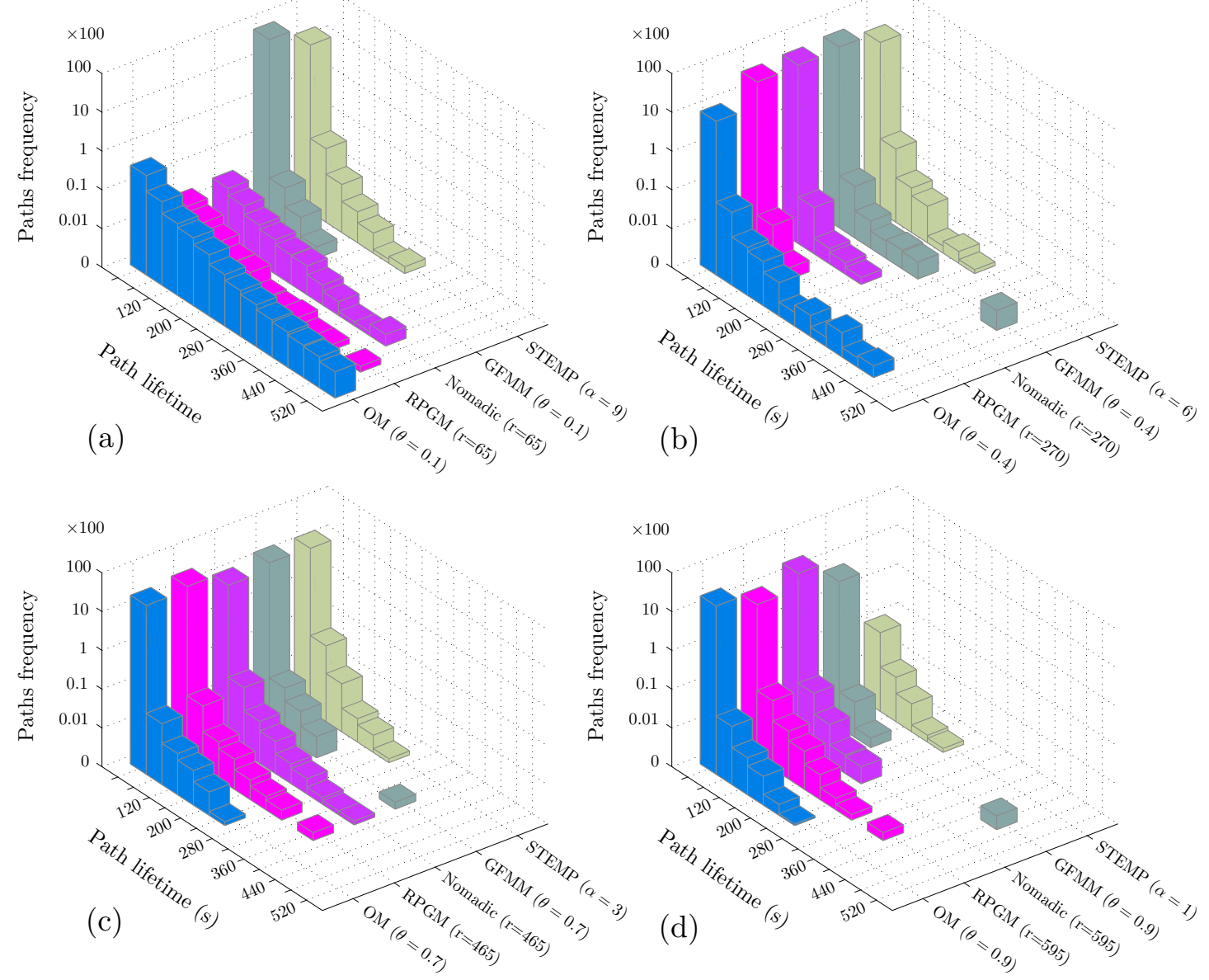

Fig. 14. Comparative evaluation between different mobility models in terms of the path lifetime distributions. OM denotes our model.

This keeps the stability of links between nodes such that the network topology is effectively static. Under high enough values of perturbation factors of network topology, the link quality is more unstable, and thus the probability of transmission failure increases, thereby increasing the packet loss probability. Therefore, the appearance of disconnected network segments Fig. 15c is particularly due to degradation of the cohesion behavior between nodes. On other hand, it could be also noticed that both RPGM and Nomadic outperform our model in terms of packet delivery and path lifetimes (see Fig. 14c). This is because the topology connectivity in the case of our model depends on the dynamic of neighbors, whereas in the case of RPGM or Nomadic the lead point has a significant impact on each group member. This leads to keeping the stability and the availability of communication links for a long time as shown in (see Fig. 14c). However, both RPGM and Nomadic depict a high frequency of isolated nodes, where the probability to get large group size is very small (see Fig. 15c). Finally, under very high values of perturbation factors, all the mobility models lead to a worst performance degradation. This is due principally to the fact that the nodes move in a highly unpredictable manner with randomly chosen speed and direction within the network area. Therefore, unexpected network segmentation may occur as the network topology and link capacities dynamically change over the time as shown in (Fig. 15d and Fig. 14d).

From the obtained results, we conclude that our model displays a realistic behavior compared with other mobility models. Only the nearby nodes to the leader are directly affected by its trajectory, whereas the others nodes are affected by their neighbors as in the real world. On the contrary, under RPGM and 

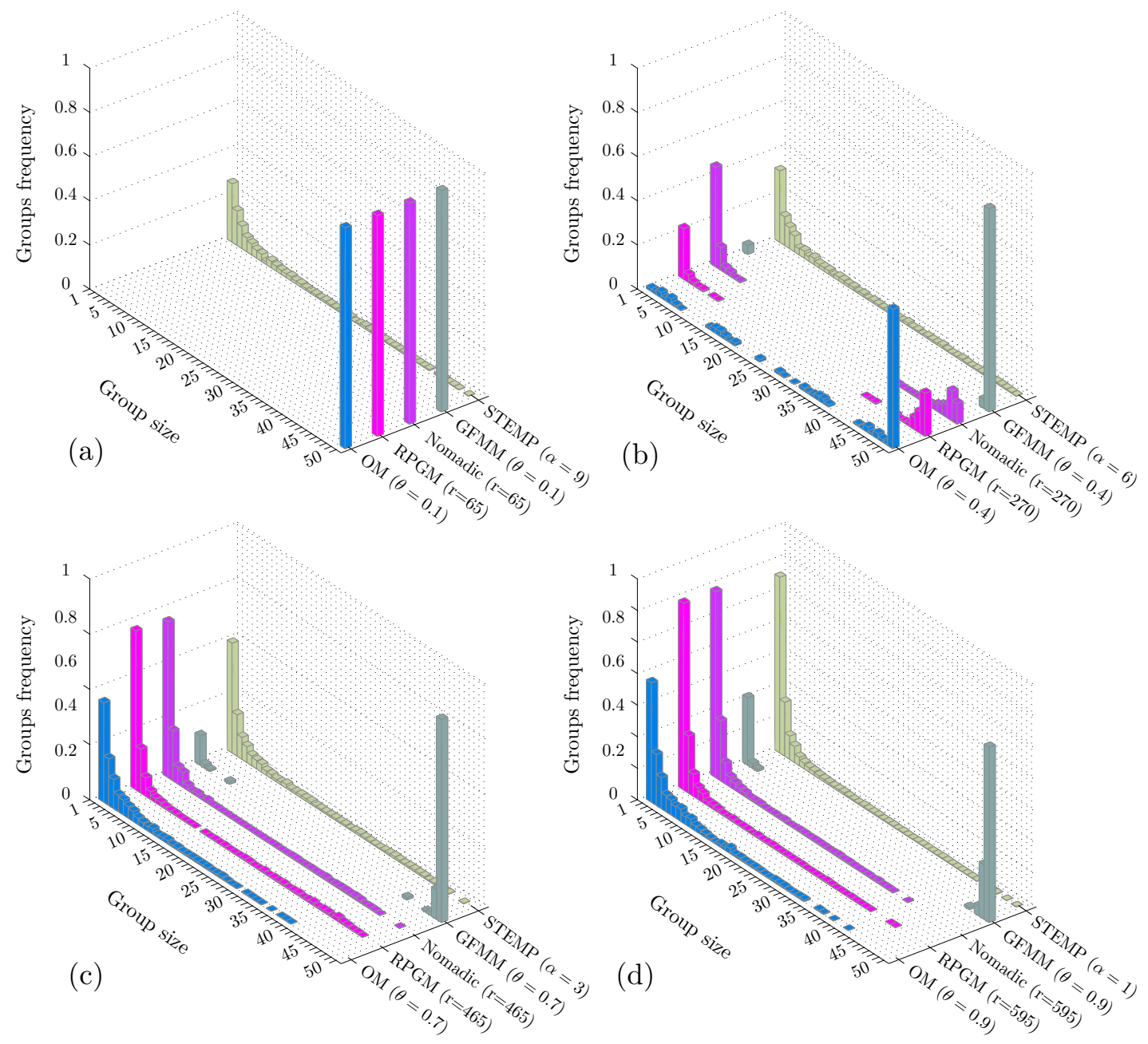

Fig. 15. Comparative evaluation between different mobility models in terms of the group size distributions.

Nomadic, every node is affected by the lead point considered as a leader member. GFMM do not support repulsion between the neighbors within the same group which is considered as a natural human behavior. Under both SLAW and STAMP, the nodes can move freely according to a random mobility model without considering some aspects of cohesion or collective motion behavior.

\section{Conclusions}

We have presented several computational statistics on the reliability of the battlefield collective motion model for dismounted soldier groups moving with or without enemy presence, including path lifetime, path lengths, packet delivery ratio, throughput and packet loss. Simulations demonstrate the effectiveness of collective motion for more reliable paths and improved stability of network topology. However, noise strongly affects the network topology state, causing partitioning and nodes dispersion. More interesting are the effects of simultaneously increasing soldiers' noise and initial velocity. Then, the network's global state is severely affected, leading to segmentation into isolated and small group nodes, and in turn to a strong degradation of communication channels. The enemies' presence means the throughput of packets received by the commander decreases as the enemy numbers increase due to limitations in terms of relay nodes and insufficient link quality, which are mainly caused by the high dynamic of soldiers. This model's advantage 
is that it allows members to make their autonomous mobility based on behavioral rules. Importantly, this allows natural (real-world) selection of rules based only on local information. This model can also simulate two different member groups (soldiers and enemies), where interactions between members of the same group are based on a collaborative approach. With very simple behavioral rules, our model can be extended to simulate more critical real-world situations. For example, it can incorporate soldiers' cognitive abilities based on memory and real-time interaction with instructions, where the soldier may be able to make a tactical battlefield decision.

Finally, simulations show that the effectiveness of the network communication depends on dismounted soldier' capability for intra-group coherence. This task, not easy in delicate battlefield situations, suggests that an adaptive and intelligent strategy for maintaining network topology might be beneficial. Another study, of whether the army's mobility improves energy consumption, will be reported in future.

\section{References}

[1] S. L. Cotton, W. G. Scanlon, E. Skafidas, B. K. Madahar, et al., Millimeter-wave stealth radio for special operations forces, Microwave Journal 53 (8) (2010) 6 .

[2] N. R. Council, Energy-Efficient Technologies for the Dismounted Soldier, The National Academies Press, ISBN 978-0309-05934-3, URL http://www.nap.edu/catalog/5905/energy-efficient-technologies-for-the-dismounted-soldier, 1997.

[3] T. J. Thomas, The Defence and National Security Capability Reporter Vol 28 Nos 04/05 of 2009 (48) (2009) 1-48.

[4] X. Hong, M. Gerla, G. Pei, C.-C. Chiang, A group mobility model for ad hoc wireless networks, in: Proceedings of the 2nd ACM international workshop on Modeling, analysis and simulation of wireless and mobile systems, ACM, 53-60, 1999.

[5] S. A. Williams, D. Huang, Group force mobility model and its obstacle avoidance capability, Acta Astronautica 65 (7) (2009) 949-957.

[6] K. H. Wang, B. Li, Group mobility and partition prediction in wireless ad-hoc networks, in: Communications, 2002. ICC 2002. IEEE International Conference on, vol. 2, IEEE, 1017-1021, 2002.

[7] K. Blakely, B. Lowekamp, A structured group mobility model for the simulation of mobile ad hoc networks, in: Proceedings of the second international workshop on Mobility management and wireless access protocols, ACM, 111-118, 2004.

[8] L. Ning, G. Yan, D. Chao, W. Jinlong, Diamond group mobility model for ad hoc network in military, in: Signal Processing, 2008. ICSP 2008. 9th International Conference on, IEEE, 2754-2756, 2008.

[9] J. M. Ng, Y. Zhang, A mobility model with group partitioning for wireless ad hoc networks, in: Information Technology and Applications, 2005. ICITA 2005. Third International Conference on, vol. 2, IEEE, 289-294, 2005.

[10] B. Zhou, K. Xu, M. Gerla, Group and swarm mobility models for ad hoc network scenarios using virtual tracks, in: Military Communications Conference, 2004. MILCOM 2004. 2004 IEEE, vol. 1, IEEE, 289-294, 2004.

[11] A. Fongen, M. Gjellerud, E. Winjum, A military mobility model for manet research, Parallel and Distributed Computing and Networks (PDCN 2009), February 16 (2009) 18.

[12] N. Vastardis, K. Yang, An enhanced community-based mobility model for distributed mobile social networks, Journal of Ambient Intelligence and Humanized Computing 5 (1) (2014) 65-75.

[13] S. Misra, P. Agarwal, Bio-inspired group mobility model for mobile ad hoc networks based on bird-flocking behavior, Soft Computing 16 (3) (2012) 437-450.

[14] A.-D. Nguyen, P. Senac, M. Diaz, Modelling mobile opportunistic networks-From mobility to structural and behavioural analysis, Ad Hoc Networks 24 (2015) 161-174.

[15] C. Zhao, M. L. Sichitiu, I. Rhee, N-body: A social mobility model with support for larger populations, Ad Hoc Networks 25 (2015) 185-196.

[16] K. H. Wang, B. Li, Efficient and guaranteed service coverage in partitionable mobile ad-hoc networks, in: INFOCOM 2002. Twenty-First Annual Joint Conference of the IEEE Computer and Communications Societies. Proceedings. IEEE, vol. 2, IEEE, 1089-1098, 2002.

[17] G. Karumanchi, S. Muralidharan, R. Prakash, Information dissemination in partitionable mobile ad hoc networks, in: Reliable Distributed Systems, 1999. Proceedings of the 18th IEEE Symposium on, IEEE, 4-13, 1999.

[18] R. Wattenhofer, L. Li, P. Bahl, Y.-M. Wang, Distributed topology control for power efficient operation in multihop wireless ad hoc networks, in: INFOCOM 2001. Twentieth annual joint conference of the IEEE computer and communications societies. Proceedings. IEEE, vol. 3, IEEE, 1388-1397, 2001.

[19] Z. Mi, Y. Yang, G. Liu, HERO: A hybrid connectivity restoration framework for mobile multi-agent networks, in: Robotics and Automation (ICRA), 2011 IEEE International Conference on, IEEE, 1702-1707, 2011.

[20] A. Konak, G. E. Buchert, J. Juro, A flocking-based approach to maintain connectivity in mobile wireless ad hoc networks, Applied Soft Computing 13 (2) (2013) 1284-1291.

[21] D. Manickavelu, R. U. Vaidyanathan, Particle swarm optimization (PSO)-based node and link lifetime prediction algorithm for route recovery in MANET, EURASIP Journal on Wireless Communications and Networking 2014 (1) (2014) 1-10.

[22] D. Vodnala, S. Aluvala, S. P. Kumar, A Backbone based multicast routing protocol for route recovery in MANETs, in: Electronics and Communication Systems (ICECS), 2015 2nd International Conference on, IEEE, 237-241, 2015.

[23] A. Zadin, T. Fevens, Maintaining path stability with node failure in mobile ad hoc networks, Procedia Computer Science 19 (2013) 1068-1073. 
[24] R. S. Abujassar, Mitigation fault of node mobility for the MANET networks by constructing a backup path with loop free: enhance the recovery mechanism for pro-active MANET protocol, Wireless Networks 22 (1) (2016) 119-133.

[25] C. W. Reynolds, Flocks, herds and schools: A distributed behavioral model, ACM Siggraph Computer Graphics 21 (4) (1987) 25-34

[26] I. D. Couzin, J. Krause, R. James, G. D. Ruxton, N. R. Franks, Collective memory and spatial sorting in animal groups, Journal of theoretical biology 218 (1) (2002) 1-11.

[27] T. Vicsek, A. Czirók, E. Ben-Jacob, I. Cohen, O. Shochet, Novel type of phase transition in a system of self-driven particles, Physical review letters 75 (6) (1995) 1226.

[28] M. Sánchez, P. Manzoni, ANEJOS: a java based simulator for ad hoc networks, Future generation computer systems 17 (5) (2001) 573-583.

[29] K. Lee, S. Hong, S. J. Kim, I. Rhee, S. Chong, Slaw: A new mobility model for human walks, in: INFOCOM 2009, IEEE, IEEE, 855-863, 2009.

Younes Regragui is a Ph.D. student in the department of Computer Science, Chouaib Doukkali University, Morocco. He got his master degrees in Computer Science from the Chouaib Doukkali University in 2013. His current research interests include the coverage problems in wireless networks, mobile and vehicular ad hoc networks.

Najem Moussa is a professor in the Department of Computer Science, Chouaib Doukkali University, Morocco. His research currently focuses on wireless networks and traffic systems. 